\title{
An improvement method for selecting the best alternative in Decision Making
}

\author{
Bin Zhou, Zheng Pei, Xinzi Ma \\ Center for Radio Administration \& Technology Development, Xihua University, \\ Chengdu, Sichuan, 610039, China \\ E-mail:pqyz@263.net
}

Received 9 April 2013

Accepted 29 August 2013

\begin{abstract}
Multiple attributes group decision making problems aim to find the best alternative for the experts from a solution set of alternatives. Because the attribute value and decision-makers evaluation with respect to the alternatives are usually vague and imprecise, fuzzy multiple attributes group decision making have been widely investigated, in which, ordering fuzzy evaluation results in fuzzy decision making is an important method to find the best alternative for the experts, difference fuzzy expressions for evaluation in fuzzy decision making problems correspond with difference aggregation operator and ranking method. In this paper, we analyze some algebraic properties of a kind of ranking method in fuzzy multiple attributes group decision-making, and prove that the ranking method is pre-ordering, its'drawback in fuzzy decision making is no unique alternative to be best alternative. Then, we provide an equivalence relation on fuzzy evaluation values based on the ranking method, and propose a linearly ordering on equivalence classes of fuzzy evaluation values. Based on the linearly ordering, we propose an improve method to handle fuzzy multiple attributes group decision-making when its ordering is pre-ordering. Some numerical examples illustrate that our method can be used to improve the best alternative of fuzzy decision making when its ordering is pre-ordering.
\end{abstract}

Keywords: Fuzzy multiple attributes group decision making, Aggregation operator, Ordering, Equivalence relation

\section{Introduction}

In many cases, decision making problems must deal with vague and imprecise information that usually involves uncertainty in their decision making frameworks 9,11,13,19. Different proposals to tackle and manage the uncertainty have been developed $15,16,17,22$, such as fuzzy sets and its' extensions, interval-valued fuzzy set, intuitionistic fuzzy set and interval-valued intuitionistic fuzzy set $1,6,7,18,23,27,28,29$. For their advantage of coping with more imprecise information, fuzzy sets and its' extensions have been adequately applied in various fields, particularly in decision-making ${ }^{2,5,11}$.
In decision making analysis, the problems are associated with: (1) The choice of expressions for evaluation in a decision making problem; (2) The choice of the aggregation operator of evaluation values of attributes in the decision making; (3) The choice of the best alternatives. In the above mentioned three steps, the aim of (1) consists of establishing the suitable formal framework or expression domain with a view to provide the performance values in uncertain environment of decision making. In practice, fuzzy numbers or an ordered structure of linguistic values can be used to explain their semantic ${ }^{8,14}$. The aim of (2) is to carry out the aggregation of evaluation values, there are many numeric 
or linguistic aggregation operators $3,4,20,21,24,25,26$ to process them. The aim of (3) consists of obtaining a collective performance value over each alternative and finding a solution set of alternatives. The solution set of alternatives is the best alternative that is the most satisfied alternative for the experts.

Generally, ordering is an important method to find the best alternative for the experts. In decision making using the 2-tuple fuzzy linguistic representation model ${ }^{8}$, i.e., let $S=\left\{s_{0}, s_{1}, \cdots, s_{g}\right\}$ be a set of linguistic term set and $\beta \in[0, g]$ a value supporting the result of a symbolic aggregation operation. Then the linguistic 2-tuple that expresses the equivalent information to $\beta$ is obtained with the function $\Delta:[0, g] \rightarrow S \times[-0.5,0.5)$ such that $\triangle(\beta)=\left(s_{i}, \alpha\right)$ with $i=\operatorname{round}(\beta)$ and $\alpha=\beta-i \in[-0.5,0.5)$, where $s_{i}$ has the closest index label to $\beta$ and $\alpha$ is the value of the symbolic translation, $\operatorname{round}(\cdot)$ is the usual rounding operation, ordering of linguistic information is processed by the linear ordered structure of linguistic values, and its natural number indexes is used to explain the ordering, which can be expressed as follows: for any 2-tuple linguistic values $\left(s_{i}, \alpha_{i}\right)$ and $\left(s_{j}, \alpha_{j}\right),\left(s_{i}, \alpha_{i}\right) \leqslant\left(s_{j}, \alpha_{j}\right)$ if and only if $\Delta^{-1}\left(s_{i}, \alpha_{i}\right)=i+\alpha_{i} \leqslant \Delta^{-1}\left(s_{j}, \alpha_{j}\right)=j+\alpha_{j}$. Based on the above mentioned ordering, any decision results represented by 2-tuple linguistic values is ordered, and the best alternative for the experts can be selected. In decision making using Atanassov's intuitionistic fuzzy sets, Li developed a methodology for solving multiattribute decision making problems based on intuitionistic fuzzy sets ${ }^{12}$, in which, decision results are represented by intuitionistic fuzzy sets, and ordering is depended on score and accuracy functions, i.e., let $C=\left\langle\mu_{C}, v_{C}\right\rangle$ be an intuitionistic fuzzy set, then the score function $s$ and the accuracy function $a$ of $C$ may be expressed by $s(C)=\mu_{C}-v_{C}$ and $a(C)=\mu_{C}+v_{C}$. Ranking two intuitionistic fuzzy sets $B=\left\langle\mu_{B}, v_{B}\right\rangle$ and $C=\left\langle\mu_{C}, v_{C}\right\rangle$ is as follows ${ }^{10}$ :

1. If $s(B)>s(C)$, then $B>C$;

2. If $s(B)=s(C)$, then

(a) If $a(B)=a(C)$, then $B=C$;

(b) If $a(B)<a(C)$, then $B<C$; (c) If $a(B)>a(C)$, then $B>C$.

Recently, Chen and Niou proposed a method for fuzzy multiple attributes group decision-making ${ }^{3}$, in which, evaluation values and decision results are represented by fuzzy sets on a finite and ordered linguistic term set $U$ called initial evaluation linguistic values, ranking decision results $\widetilde{P}_{1}$ and $\widetilde{P}_{2}$ represented by fuzzy sets on $U$ is depended on the score $S\left(\widetilde{P}_{1} \ominus \widetilde{P}_{2}\right)$ of the weighted difference of their membership values.

A bird's eye view in the recent specialized literature about decision making problems, ranking methods are an important aspect to select the best alternative for the experts, difference expressions for evaluation in a decision making problem correspond with difference aggregation operator and ranking method. From the algebraic point of view, ranking methods is to order decision results to obtain the best alternative for the experts, some of them is linearly ordered, the others is pre-ordering. Theoretically, there is no unique alternative to be best in pre-ordered set, this is drawback of decision making approach when its ordering is pre-ordering. In this paper, we aim to analyze some algebraic properties of the score proposed in ${ }^{3}$. Then we provide an equivalence relation on decision results to order aggregation values in decision making, the new ordering method can overcome drawback of decision making approach when its ordering is pre-ordering, example shows that the method is an alternative decision making method when its ordering is pre-ordering. The paper is organized as follows: In Section 2, we briefly review the decision making method and the score proposed in ${ }^{3}$. In Section 3, we analyze some algebraic properties of the score. In Section 4, we devote to discuss an equivalence relation on fuzzy sets based on the score and provide a new method to handle multi-criteria decision-making when its ordering is pre-ordering. In Section 5, we use two examples to illustrate the proposed method. We conclude in Section 6.

\section{Preliminaries}

There are different approaches to select linguistic descriptors and different ways to define their semantics in fuzzy multi-criteria decision making prob- 
lems. The selection of linguistic descriptors in ${ }^{3}$ can be performed as follows: Assume that $U=$ $\left\{s_{-m}, \cdots, s_{0}, \cdots, s_{m}\right\}$ is a finite and ordered linguistic term set, and linguistic descriptors are fuzzy sets on $U, e . g ., \widetilde{P}_{1}=a_{1} / s_{B_{1}}+a_{2} / s_{B_{2}}+\cdots+a_{n} / s_{B_{n}}$ and $\widetilde{P}_{2}=b_{1} / s_{B_{1}}+b_{2} / s_{B_{2}}+\cdots+b_{n} / s_{B_{n}}$, where $\left\{s_{B_{1}}, s_{B_{2}}, \cdots, s_{B_{n}}\right\} \subseteq U$, integer values $B_{1}<B_{2}<$ $\cdots<B_{n}$ and $s_{B_{1}}<s_{B_{2}}<\cdots<s_{B_{n}}, a_{i} \in[0,1]$ denotes the grade of membership of $s_{B_{i}}$ in the fuzzy set $\widetilde{P}_{1}$, $b_{j} \in[0,1]$ denotes the grade of membership of $s_{B_{j}}$ in the fuzzy set $\widetilde{P}_{2}, 1 \leqslant i, j \leqslant n$.

Some operations of fuzzy sets defined on $U$ are shown as follows: The addition operation between two fuzzy sets is

$$
\begin{aligned}
\widetilde{P}_{1} \oplus \widetilde{P}_{2}= & \left(a_{1} / s_{B_{1}}+\cdots+a_{n} / s_{B_{n}}\right) \oplus\left(b_{1} / s_{B_{1}}\right. \\
& \left.+\cdots+b_{n} / s_{B_{n}}\right) \\
= & \left(a_{1}+b_{1}\right) / s_{B_{1}}+\cdots+\left(a_{n}+b_{n}\right) / s_{B_{n}} .
\end{aligned}
$$

The multiplication operation between $\alpha$ and $\widetilde{P}_{1}$ is

$$
\begin{aligned}
\alpha \otimes \widetilde{P}_{1} & =\alpha \otimes\left(a_{1} / s_{B_{1}}+\cdots+a_{n} / s_{B_{n}}\right) \\
& =\left(\alpha \times a_{1}\right) / s_{B_{1}}+\cdots+\left(\alpha \times a_{n}\right) / s_{B_{n}} .
\end{aligned}
$$

Based on the above mentioned operations, FIOWA operator can be defined as follows: Let $\left\{\left\langle u_{1}, \widetilde{P}_{1}\right\rangle,\left\langle u_{2}, \widetilde{P}_{2}\right\rangle, \cdots,\left\langle u_{n}, \widetilde{P}_{n}\right\rangle\right\}$ be OWA pairs ${ }^{26}$ and $W=\left(w_{1}, w_{2}, \cdots, w_{n}\right)$ a weighting vector, where $u_{i}$ in the OWA pair $\left\langle u_{i}, \widetilde{P}_{i}\right\rangle$ is called the order inducing variable, $\widetilde{P}_{i}$ is called the uncertain linguistic argument variable, $w_{i}$ denotes the $i$ th weight such that $w_{i} \in[0,1]$ and $\sum_{i=1}^{n} w_{i}=1,1 \leqslant i \leqslant n$.

$F_{\text {FIOWA }}\left(\left\langle u_{1}, \widetilde{P}_{1}\right\rangle, \cdots,\left\langle u_{n}, \widetilde{P}_{n}\right\rangle\right)=w_{1} \widetilde{P}_{b_{1}} \oplus \cdots \oplus w_{n} \widetilde{P}_{b_{n}}$.

In which, $\widetilde{P}_{b_{j}}(1 \leqslant j \leqslant n)$ is the value of the OWA pair $\left\langle u_{i}, \widetilde{P}_{i}\right\rangle$ having the $j$ th largest order inducing $u_{i}$ value.

The score $S\left(\widetilde{P}_{1} \ominus \widetilde{P}_{2}\right)$ of the weighted difference of the membership values between $\widetilde{P}_{1}$ and $\widetilde{P}_{2}$ is

$$
\begin{aligned}
& S\left(\widetilde{P}_{1} \ominus \widetilde{P}_{2}\right)=\left(a_{1} / s_{B_{1}}+\cdots+a_{n} / s_{B_{n}}\right) \ominus\left(b_{1} / s_{B_{1}}+\cdots\right. \\
& \left.\quad+b_{n} / s_{B_{n}}\right)=B_{1} \times\left(a_{1}-b_{1}\right)+\cdots+B_{n} \times\left(a_{n}-b_{n}\right) .
\end{aligned}
$$

Formally, $S\left(\widetilde{P}_{1} \ominus \widetilde{P}_{2}\right)$ provides an order relation between two fuzzy sets on $U$ defined as follows.
Definition 1. ${ }^{3}$ Let $\widetilde{P}_{1}, \widetilde{P}_{2}$ and $\widetilde{P}_{3}$ be three fuzzy sets in the universe of discourse $U$, where $U=$ $\left\{s_{B_{1}}, s_{B_{2}}, \cdots, s_{B_{n}}\right\}$.

1. If $S\left(\widetilde{P}_{1} \ominus \widetilde{P}_{1}\right) \geqslant S\left(\widetilde{P}_{1} \ominus \widetilde{P}_{2}\right)$, then $\widetilde{P}_{1} \leqslant \widetilde{P}_{2}$;

2. If $S\left(\widetilde{P}_{1} \ominus \widetilde{P}_{2}\right) \geqslant S\left(\widetilde{P}_{1} \ominus \widetilde{P}_{3}\right)$, then $\widetilde{P}_{2} \leqslant \widetilde{P}_{3}$;

3. If $S\left(\widetilde{P}_{1} \ominus \widetilde{P}_{1}\right) \geqslant S\left(\widetilde{P}_{1} \ominus \widetilde{P}_{2}\right)$ and $S\left(\widetilde{P}_{1} \ominus \widetilde{P}_{2}\right) \geqslant$ $S\left(\widetilde{P}_{1} \ominus \widetilde{P}_{3}\right)$, then $\widetilde{P}_{1} \leqslant \widetilde{P}_{2} \leqslant \widetilde{P}_{3}$.

Based on FIOWA operator and the score $S\left(\widetilde{P}_{i} \ominus\right.$ $\widetilde{P}_{j}$ ) between fuzzy sets $\widetilde{P}_{i}$ and $\widetilde{P}_{j}$, the proposed method for fuzzy multiple attributes group decisionmaking based on FIOWA operators is as follows: Assume that there are $n$ alternatives $\left\{x_{1}, x_{2}, \cdots, x_{n}\right\}$, $m$ attributes $\left\{f_{1}, f_{2}, \cdots, f_{m}\right\}$ and $g$ decision-makers $\left\{D_{1}, D_{2}, \cdots, D_{g}\right\}$. Let $H=\left[h_{1}, h_{2}, \cdots, h_{g}\right]^{T}$ be the weighting vector of the decision-makers, where $h_{k}$ denotes the weight of decision-maker $D_{k}, 1 \leqslant k \leqslant g$ and $\sum_{k=1}^{g} h_{k}=1$. Let $V=\left[v_{1}, v_{2}, \cdots, v_{m}\right]^{T}$ be the weighting vector of the attributes, where $v_{i}$ denotes the weight of attribute $f_{i}, 1 \leqslant i \leqslant m$ and $\sum_{i=1}^{m} v_{i}=1$.

The fuzzy evaluating matrix $\widetilde{F}_{k}$ for decisionmaker $D_{k}$ with respect to attributes of alternatives is as follows:

$$
\widetilde{F}_{k}=\begin{aligned}
& f_{1} \\
& f_{2} \\
& \vdots \\
& f_{m}
\end{aligned}\left(\begin{array}{cccc}
\frac{x_{1}}{f_{11}^{k}} & \frac{x_{2}}{f_{12}^{k}} & \cdots & \frac{x_{n}}{f_{21}^{k}} \\
f_{22}^{k} & \cdots & \widetilde{f_{2 n}^{k}} \\
\vdots & \vdots & \vdots & \vdots \\
f_{m 1}^{k} & f_{m 2}^{k} & \cdots & \frac{f_{m n}^{k}}{k}
\end{array}\right) .
$$

In which, every $\widetilde{f_{i j}^{k}}$ is a fuzzy set on linguistic term set $U, 1 \leqslant k \leqslant g$.

By using FIOWA operator, the fuzzy evaluating value of all decision-makers with respect to the attribute $f_{i}$ of the alternative $x_{j}$ is as follows

$$
\begin{aligned}
\widetilde{Z_{i j}} & =F_{F I O W A}\left(\left\langle h_{1}, \widetilde{f_{i j}^{1}}\right\rangle, \cdots,\left\langle h_{k}, \widetilde{f_{i j}^{k}}\right\rangle\right) \\
& =w_{1} \widetilde{f_{i j}^{\prime}} \oplus \cdots \oplus w_{k} \widetilde{f_{i j}^{\prime k}} .
\end{aligned}
$$

In which, $W=\left(w_{1}, w_{2}, \cdots, w_{k}\right)$ is a weighting vector of $\left\{\widetilde{f_{i j}^{1}}, \widetilde{f_{i j}^{2}}, \cdots, \widetilde{f_{i j}^{k}}\right\}$. 
The score $\widetilde{E}_{j}$ of each alternative $x_{j}$ represented by fuzzy set on $U$ is as follows:

$$
\begin{aligned}
\widetilde{E_{j}} & =F_{\text {FIOWA }}\left(\left\langle v_{1}, \widetilde{Z_{1 j}}\right\rangle, \cdots,\left\langle v_{m}, \widetilde{Z_{m j}}\right\rangle\right) \\
& =r_{1} \widetilde{Z_{1 j}} \oplus r_{2}{\widetilde{Z_{2 j}}}^{\prime} \oplus \cdots \oplus r_{m} \widetilde{Z_{m j}} .
\end{aligned}
$$

In which, $\left\langle v_{i}, \widetilde{Z_{i j}}\right\rangle$ is the OWA pair, $R=$ $\left(r_{1}, r_{2}, \cdots, r_{m}\right)$ is a weighting vector of $\left\{\widetilde{Z_{1 j}}, \widetilde{Z_{2 j}}, \cdots, \widetilde{Z_{m j}}\right\}, 1 \leqslant i \leqslant m, 1 \leqslant j \leqslant n$.

To obtain the better alternative as the final decision, the score of $S\left(\widetilde{E_{j_{1}}} \ominus \widetilde{E_{j_{2}}}\right)$ for any $j_{1}$ and $j_{2}$ is needed, the smaller the value of $S\left(\widetilde{E_{j_{1}}} \ominus \widetilde{E_{j_{2}}}\right)$ is , the better alternative $x_{j_{2}}$ is.

Example 1. Let $U=\left\{s_{-4}=\right.$ extremely poor,$s_{-3}=$ very poor, $s_{-2}=$ poor,$s_{-1}=$ slightly poor $s_{0}=$ fair, $s_{1}=$ slightly good, $s_{2}=$ good, $s_{3}=$ very good, $s_{4}=$ extremely good $\}, \quad \widetilde{P}_{1}, \widetilde{P}_{2}, \widetilde{P}_{3}$ and $\widetilde{P}_{4}$ be the score of alternatives $x_{j} \quad(1 \leqslant j \leqslant$ 4), which are fuzzy sets on $U$, i.e., $\widetilde{P}_{1}=$ $0.045 / s_{-1}+0.165 / s_{0}+0.4 / s_{1}+0.25 / s_{2}+0.14 / s_{3}$, $\widetilde{P}_{2}=0.14 / s_{0}+0.17 / s_{1}+0.315 / s_{2}+0.285 / s_{3}+$ $0.09 / s_{4}, \widetilde{P}_{3}=0.08 / s_{-2}+0.06 / s_{-1}+0.225 / s_{0}+$ $0.35 / s_{1}+0.14 / s_{2}+0.1 / s_{3}+0.045 / s_{4}$, and $\widetilde{P}_{4}=$ $0.1 / s_{-1}+0.165 / s_{0}+0.275 / s_{1}+0.195 / s_{2}+$ $0.2 / s_{3}+0.065 / s_{4}$. According to (1), we have $S\left(\widetilde{P}_{1} \ominus \widetilde{P}_{1}\right)=S\left(\left(0.045 / s_{-1}+0.165 / s_{0}+0.4 / s_{1}+\right.\right.$ $\left.0.25 / s_{2}+0.14 / s_{3}\right) \ominus\left(0.045 / s_{-1}+0.165 / s_{0}+\right.$ $\left.\left.0.4 / s_{1}+0.25 / s_{2}+0.14 / s_{3}\right)\right)=(-1) \times(0.045-$ $0.045)+0 \times(0.165-0.165)+1 \times(0.4-0.4)+$ $2 \times(0.25-0.25)+3 \times(0.14-0.14))=0$. Similarly, $S\left(\widetilde{P}_{1} \ominus \widetilde{P}_{2}\right)=-0.74, S\left(\widetilde{P}_{1} \ominus \widetilde{P}_{3}\right)=0.37$ and $S\left(\widetilde{P}_{1} \ominus \widetilde{P}_{4}\right)=-0.15$.

Due to $S\left(\widetilde{P}_{1} \ominus \widetilde{P}_{1}\right) \geqslant S\left(\widetilde{P}_{1} \ominus \widetilde{P}_{2}\right), S\left(\widetilde{P}_{1} \ominus \widetilde{P}_{3}\right) \geqslant$ $S\left(\widetilde{P}_{1} \ominus \widetilde{P}_{1}\right)$ and $S\left(\widetilde{P}_{1} \ominus \widetilde{P}_{1}\right) \geqslant S\left(\widetilde{P}_{1} \ominus \widetilde{P}_{4}\right)$, we have $\widetilde{P}_{1} \leqslant \widetilde{P}_{2}, \widetilde{P}_{3} \leqslant \widetilde{P}_{1}$ and $\widetilde{P}_{1} \leqslant \widetilde{P}_{4}$. On the other hand, $S\left(\widetilde{P}_{1} \ominus \widetilde{P}_{2}\right) \leqslant S\left(\widetilde{P}_{1} \ominus \widetilde{P}_{4}\right)$, we have $\widetilde{P}_{4} \leqslant \widetilde{P}_{2}$. Finally, we have $\widetilde{P}_{3} \leqslant \widetilde{P}_{1} \leqslant \widetilde{P}_{4} \leqslant \widetilde{P}_{2}$, i.e., $x_{2}$ is the best alternative.

\section{Properties of the score}

In this section, we analyze some algebraic properties of the score $S\left(\widetilde{P}_{i} \ominus \widetilde{P}_{j}\right)$. We show that the order relation decided by $S\left(\widetilde{P}_{1} \ominus \widetilde{P}_{2}\right)$ is a pre-order relation of fuzzy sets on $U$.
Proposition 1. For any fuzzy sets $\widetilde{P}_{i}, \widetilde{P}_{j}$ and $\widetilde{P}_{k}$ on $U=\left\{s_{B_{1}}, s_{B_{2}}, \cdots, s_{B_{n}}\right\}$.

1. $S\left(\widetilde{P}_{i} \ominus \widetilde{P}_{i}\right)=0$;

2. $S\left(\widetilde{P}_{i} \ominus \widetilde{P}_{j}\right)=-S\left(\widetilde{P}_{j} \ominus \widetilde{P}_{i}\right)$;

3. $S\left(\widetilde{P}_{i} \ominus \widetilde{P}_{k}\right)=S\left(\widetilde{P}_{i} \ominus \widetilde{P}_{j}\right)+S\left(\widetilde{P}_{j} \ominus \widetilde{P}_{k}\right)$;

4. If $S\left(\widetilde{P}_{i} \ominus \widetilde{P}_{j}\right) \geqslant 0$ and $S\left(\widetilde{P}_{j} \ominus \widetilde{P}_{k}\right) \geqslant 0$, then $S\left(\widetilde{P}_{i} \ominus \widetilde{P}_{k}\right) \geqslant 0$.

Proof. Let $\widetilde{P}_{i}=c_{1} / s_{B_{1}}+c_{2} / s_{B_{2}}+\cdots+c_{n} / s_{B_{n}}, \widetilde{P}_{j}=$ $d_{1} / s_{B_{1}}+d_{2} / s_{B_{2}}+\cdots+d_{n} / s_{B_{n}}$ and $\widetilde{P}_{k}=e_{1} / s_{B_{1}}+$ $e_{2} / s_{B_{2}}+\cdots+e_{n} / s_{B_{n}}$.

1. According to (1), $S\left(\widetilde{P}_{i} \ominus \widetilde{P}_{i}\right)=0$ is obvious;

2. $S\left(\widetilde{P}_{i} \ominus \widetilde{P}_{j}\right)=B_{1} \times\left(c_{1}-d_{1}\right)+B_{2} \times\left(c_{2}-d_{2}\right)+$ $\cdots+B_{n} \times\left(c_{n}-d_{n}\right)=-\left[B_{1} \times\left(d_{1}-c_{1}\right)+B_{2} \times\right.$ $\left.\left(d_{2}-c_{2}\right)+\cdots+B_{n} \times\left(d_{n}-c_{n}\right)\right]=-S\left(\widetilde{P}_{j} \ominus \widetilde{P}_{i}\right) ;$

3. Because $S\left(\widetilde{P}_{i} \ominus \widetilde{P}_{j}\right)=B_{1} \times\left(c_{1}-d_{1}\right)+B_{2} \times$ $\left(c_{2}-d_{2}\right)+\cdots+B_{n} \times\left(c_{n}-d_{n}\right)$ and $S\left(\widetilde{P}_{j} \ominus\right.$ $\left.\widetilde{P}_{k}\right)=B_{1} \times\left(d_{1}-e_{1}\right)+B_{2} \times\left(d_{2}-e_{2}\right)+\cdots+$ $B_{n} \times\left(d_{n}-e_{n}\right)$. Hence, $S\left(\widetilde{P}_{i} \ominus \widetilde{P}_{j}\right)+S\left(\widetilde{P}_{j} \ominus\right.$ $\left.\widetilde{P}_{k}\right)=\left[B_{1} \times\left(c_{1}-d_{1}\right)+B_{2} \times\left(c_{2}-d_{2}\right)+\cdots+\right.$ $\left.B_{n} \times\left(c_{n}-d_{n}\right)\right]+\left[B_{1} \times\left(d_{1}-e_{1}\right)+B_{2} \times\left(d_{2}-\right.\right.$ $\left.\left.e_{2}\right)+\cdots+B_{n} \times\left(d_{n}-e_{n}\right)\right]=\left[B_{1} \times\left(c_{1}-d_{1}\right)+\right.$ $\left.B_{1} \times\left(d_{1}-e_{1}\right)\right]+\left[B_{2} \times\left(c_{2}-d_{2}\right)+B_{2} \times\left(d_{2}-\right.\right.$ $\left.\left.e_{2}\right)\right]+\cdots+\left[B_{n} \times\left(c_{n}-d_{n}\right)+B_{n} \times\left(d_{n}-e_{n}\right)\right]=$ $B_{1} \times\left(c_{1}-e_{1}\right)+B_{2} \times\left(c_{2}-e_{2}\right)+\cdots+B_{n} \times$ $\left(c_{n}-e_{n}\right)=S\left(\widetilde{P}_{i} \ominus \widetilde{P}_{k}\right) ;$

4. If $S\left(\widetilde{P}_{i} \ominus \widetilde{P}_{j}\right) \geqslant 0$ and $S\left(\widetilde{P}_{j} \ominus \widetilde{P}_{k}\right) \geqslant 0$, according to (3), $S\left(\widetilde{P}_{i} \ominus \widetilde{P}_{k}\right)=S\left(\widetilde{P}_{i} \ominus \widetilde{P}_{j}\right)+S\left(\widetilde{P}_{j} \ominus \widetilde{P}_{k}\right) \geqslant 0$.

In the rest of the paper, $\widetilde{P}_{i} \leqslant \widetilde{P}_{j}$ is denoted by $\widetilde{P}_{i} \leqslant s \widetilde{P}_{j}$. Based on Proposition 1, Definition 1 can be modified as follows.

Definition 2. For any two fuzzy sets $\widetilde{P}_{i}$ and $\widetilde{P}_{j}$ on $U=\left\{s_{B_{1}}, s_{B_{2}}, \cdots, s_{B_{n}}\right\}, \widetilde{P}_{i} \leqslant s \widetilde{P}_{j}$ if and only if $S\left(\widetilde{P}_{i} \ominus \widetilde{P}_{j}\right) \leqslant 0$.

According to Definition 2, 1) if $S\left(\widetilde{P}_{1} \ominus \widetilde{P}_{1}\right) \geqslant$ $S\left(\widetilde{P}_{1} \ominus \widetilde{P}_{2}\right)$, i.e., $0 \geqslant S\left(\widetilde{P}_{1} \ominus \widetilde{P}_{2}\right)$, then $\left.\widetilde{P}_{1} \leqslant s \widetilde{P}_{2} ; 2\right)$ If $S\left(\widetilde{P}_{1} \ominus \widetilde{P}_{2}\right) \geqslant S\left(\widetilde{P}_{1} \ominus \widetilde{P}_{3}\right)$, i.e., $S\left(\widetilde{P}_{1} \ominus \widetilde{P}_{3}\right)-S\left(\widetilde{P}_{1} \ominus\right.$ 
$\left.\widetilde{P}_{2}\right) \leqslant 0$, according to Proposition 1(3), $S\left(\widetilde{P}_{1} \ominus \widetilde{P}_{3}\right)-$ $S\left(\widetilde{P}_{1} \ominus \widetilde{P}_{2}\right)=S\left(\widetilde{P}_{2} \ominus \widetilde{P}_{3}\right) \leqslant 0$, then $\left.\widetilde{P}_{2} \leqslant s \widetilde{P}_{3} ; 3\right)$ If $S\left(\widetilde{P}_{1} \ominus \widetilde{P}_{1}\right) \geqslant S\left(\widetilde{P}_{1} \ominus \widetilde{P}_{2}\right)$ and $S\left(\widetilde{P}_{1} \ominus \widetilde{P}_{2}\right) \geqslant S\left(\widetilde{P}_{1} \ominus \widetilde{P}_{3}\right)$, i.e., $S\left(\widetilde{P}_{1} \ominus \widetilde{P}_{2}\right) \leqslant 0$ and $S\left(\widetilde{P}_{2} \ominus \widetilde{P}_{3}\right) \leqslant 0$, hence, $\widetilde{P}_{1} \leqslant$ $\widetilde{P}_{2}$ and $\widetilde{P}_{2} \leqslant \widetilde{P}_{3}$, i.e., $\widetilde{P}_{1} \leqslant \widetilde{P}_{2} \leqslant \widetilde{P}_{3}$.

The following proposition show that " $\leqslant s$ " is a pre-order relation of fuzzy sets on $U=\left\{s_{B_{1}}\right.$, $\left.s_{B_{2}}, \cdots, s_{B_{n}}\right\}$.

Proposition 2. For any fuzzy sets $\widetilde{P}_{i}, \widetilde{P}_{j}$ and $\widetilde{P}_{k}$ on $U$, " $\leqslant_{s}$ " satisfies reflexivity and transitivity, i.e.,

\section{Reflexivity: $\widetilde{P}_{i} \leqslant s \widetilde{P}_{i}$;}

2. Transitivity: If $\widetilde{P}_{i} \leqslant s, \widetilde{P}_{j}, \widetilde{P}_{j} \leqslant s, \widetilde{P}_{k}$, then $\widetilde{P}_{i} \leqslant{ }_{s} \widetilde{P}_{k}$.

Proof. Let $\widetilde{P}_{i}=c_{1} / s_{B_{1}}+c_{2} / s_{B_{2}}+\cdots+c_{n} / s_{B_{n}}, \widetilde{P}_{j}=$ $d_{1} / s_{B_{1}}+d_{2} / s_{B_{2}}+\cdots+d_{n} / s_{B_{n}}$ and $\widetilde{P}_{k}=e_{1} / s_{B_{1}}+$ $e_{2} / s_{B_{2}}+\cdots+e_{n} / s_{B_{n}}$.

Due to $S\left(\widetilde{P}_{i} \ominus \widetilde{P}_{i}\right) \leqslant S\left(\widetilde{P}_{i} \ominus \widetilde{P}_{i}\right)=0$, reflexivity $\widetilde{P}_{i} \leqslant s \widetilde{P}_{i}$ is obvious.

Due to $\widetilde{P}_{i} \leqslant s \widetilde{P}_{j}$ if and only if $S\left(\widetilde{P}_{i} \ominus \widetilde{P}_{j}\right) \leqslant 0, \widetilde{P}_{j} \leqslant s$ $\widetilde{P}_{k}$ if and only if $S\left(\widetilde{P}_{j} \ominus \widetilde{P}_{k}\right) \leqslant 0$. According to Proposition 1 (3), $S\left(\widetilde{P}_{i} \ominus \widetilde{P}_{k}\right)=S\left(\widetilde{P}_{i} \ominus \widetilde{P}_{j}\right)+S\left(\widetilde{P}_{j} \ominus \widetilde{P}_{k}\right) \leqslant 0$, i.e., $\widetilde{P}_{i} \leqslant s \widetilde{P}_{k}$, transitivity holds.

The following example means that " $\leqslant_{s}$ " is not satisfied anti-symmetry.

Example 2. Let $\widetilde{P}_{1}, \widetilde{P}_{2}$ be two fuzzy sets on $U=$ $\left\{s_{-4}, s_{-3}, \cdots, s_{4}\right\}$, i.e., $\widetilde{P}_{1}=0.1 / s_{-1}+0.165 / s_{0}+$ $0.275 / s_{1}+0.195 / s_{2}+0.2 / s_{3}+0.065 / s_{4}, \widetilde{P}_{2}=$ $0.045 / s_{-4}+0.11 / s_{-2}+0.06 / s_{-1}+0.15 / s_{0}+$ $0.305 / s_{1}+0.4 / s_{2}+0.1 / s_{3}+0.12 / s_{4}$. According to (3), we have $S\left(\widetilde{P}_{1} \ominus \widetilde{P}_{2}\right)=S\left(\left(0.1 / s_{-1}+0.165 / s_{0}+\right.\right.$ $\left.0.275 / s_{1}+0.195 / s_{2}+0.2 / s_{3}+0.065 / s_{4}\right) \ominus$ $\left(0.045 / s_{-4}+0.11 / s_{-2}+0.06 / s_{-1}+0.15 / s_{0}+\right.$ $\left.\left.0.305 / s_{1}+0.4 / s_{2}+0.1 / s_{3}+0.12 / s_{4}\right)\right)=(-4) \times$ $(0-0.045)+(-2) \times(0-0.11)+(-1) \times(0.1-$ $0.06)+0 \times(0.165-0.15)+1 \times(0.275-0.305)+$ $2 \times(0.195-0.4)+3 \times(0.2-0.1)+4 \times(0.065-$ $0.12))=0$. Clearly, $S\left(\widetilde{P}_{1} \ominus \widetilde{P}_{2}\right)=0$ and $\widetilde{P}_{1} \neq \widetilde{P}_{2}$. Proposition 2 and Example 2 mean that " $\leqslant s$ " is not partially ordered relation.

Proposition 3. For any fuzzy sets $\widetilde{P}_{i}, \widetilde{P}_{j}$ on $U$, we always have $\widetilde{P}_{i} \leqslant s \widetilde{P}_{j}$ or $\widetilde{P}_{j} \leqslant{ }_{s} \widetilde{P}_{i}$.
Proof. For any fuzzy sets $\widetilde{P}_{i}, \widetilde{P}_{j}$ on $U$, according to (1), we can calculate $S\left(\widetilde{P}_{i} \ominus \widetilde{P}_{j}\right)$. According to Definition 1 , if $S\left(\widetilde{P}_{i} \ominus \widetilde{P}_{j}\right) \leqslant 0$, then $\widetilde{P}_{i} \leqslant s \widetilde{P}_{j}$. Else $S\left(\widetilde{P}_{i} \ominus \widetilde{P}_{j}\right) \geqslant 0$, according to Proposition 1 (2), $S\left(\widetilde{P}_{j} \ominus \widetilde{P}_{i}\right)=-S\left(\widetilde{P}_{i} \ominus \widetilde{P}_{j}\right) \leqslant 0$, i.e., $\widetilde{P}_{j} \leqslant s \widetilde{P}_{i}$.

Proposition 3 means that for any fuzzy sets $\widetilde{P}_{i}$ and $\widetilde{P}_{j}$ on $U$, if $S\left(\widetilde{P}_{j} \ominus \widetilde{P}_{k}\right) \neq 0$, then $\widetilde{P}_{i}$ and $\widetilde{P}_{j}$ is comparable, i.e., $\widetilde{P}_{i} \geqslant_{s} \widetilde{P}_{j}$ or $\widetilde{P}_{i} \leqslant s \widetilde{P}_{j}$. Proposition 2 and Proposition 3 show that we can not rank fuzzy sets $\widetilde{P}_{i}$ and $\widetilde{P}_{j}$ on $U$ when $S\left(\widetilde{P}_{i} \ominus \widetilde{P}_{j}\right)=0$.

Example 3. Let $\widetilde{P}_{1}, \widetilde{P}_{2}, \widetilde{P}_{3}$ and $\widetilde{P}_{4}$ be four scores of alternative $x_{j}, 1 \leqslant j \leqslant 4$, which are fuzzy sets on $U=$ $\left\{s_{-4}, s_{-3}, \cdots, s_{4}\right\}$, i.e., $\widetilde{P}_{1}=0.08 / s_{-2}+0.06 / s_{-1}+$ $0.225 / s_{0}+0.35 / s_{1}+0.14 / s_{2}+0.1 / s_{3}+0.045 / s_{4}$, $\widetilde{P}_{2}=0.1 / s_{-1}+0.165 / s_{0}+0.275 / s_{1}+0.195 / s_{2}+$ $0.2 / s_{3}+0.065 / s_{4}, \widetilde{P}_{3}=0.045 / s_{-4}+0.11 / s_{-2}+$ $0.06 / s_{-1}+0.15 / s_{0}+0.305 / s_{1}+0.4 / s_{2}+0.1 / s_{3}+$ $0.12 / s_{4}, \quad$ and $\widetilde{P}_{4}=0.16 / s_{-4}+0.08 / s_{-3}+$ $0.13 / s_{-1}+0.21 / s_{0}+0.28 / s_{1}+0.17 / s_{2}+0.225 / s_{4}$. According to (1), we have $S\left(\widetilde{P}_{1} \ominus \widetilde{P}_{2}\right)=-0.535$, $S\left(\widetilde{P}_{1} \ominus \widetilde{P}_{3}\right)=-0.535, S\left(\widetilde{P}_{1} \ominus \widetilde{P}_{4}\right)=0.38, S\left(\widetilde{P}_{2} \ominus\right.$ $\left.\widetilde{P}_{3}\right)=0, S\left(\widetilde{P}_{2} \ominus \widetilde{P}_{4}\right)=0.915, S\left(\widetilde{P}_{3} \ominus \widetilde{P}_{4}\right)=0.915$.

So, we have $\widetilde{P}_{4} \leqslant s \widetilde{P}_{1} \leqslant s \widetilde{P}_{2}, \widetilde{P}_{4} \leqslant s \widetilde{P}_{1} \leqslant s \widetilde{P}_{3}$, $\widetilde{P}_{2} \leqslant s \widetilde{P}_{3}$ and $\widetilde{P}_{3} \leqslant s \widetilde{P}_{2}$ due to $S\left(\widetilde{P}_{2} \ominus \widetilde{P}_{3}\right)=0$, i.e., $\widetilde{P}_{4} \leqslant s \widetilde{P}_{1} \leqslant s\left\{\widetilde{P}_{2}, \widetilde{P}_{3}\right\}$. Hence, we can not choose the better alternative from $x_{2}$ and $x_{3}$.

\section{An improved ordering method based on the score}

In this section, we discuss an equivalence relation on fuzzy sets based on the score $S\left(\widetilde{P}_{i} \ominus \widetilde{P}_{j}\right)$, and provide an improved ordering method to handle fuzzy decision making problems when $S\left(\widetilde{P}_{i} \ominus \widetilde{P}_{j}\right)=0$.

\subsection{An equivalence relation on fuzzy sets}

Definition 3. For any two fuzzy sets $\widetilde{P}_{i}$ and $\widetilde{P}_{j}$ on $U=\left\{s_{B_{1}}, s_{B_{2}}, \cdots, s_{B_{n}}\right\}, \widetilde{P}_{i} \sim_{s} \widetilde{P}_{j}$ if and only if $S\left(\widetilde{P}_{i} \ominus \widetilde{P}_{j}\right)=0$.

the following properties show that $\sim_{s}$ is an equivalence relation of fuzzy sets on $U=\left\{s_{B_{1}}\right.$, $\left.s_{B_{2}}, \cdots, s_{B_{n}}\right\}$. 
Proposition 4. For any fuzzy sets $\widetilde{P}_{i}, \widetilde{P}_{j}$ and $\widetilde{P}_{k}$ on $U$, " $\sim_{s}$ " is an equivalence relation on fuzzy sets, i.e., " $\sim_{s}$ " satisfies

\section{Reflexivity: $\widetilde{P}_{i} \sim_{s} \widetilde{P}_{i}$;}

2. Symmetry: If $\widetilde{P}_{i} \sim_{s} \widetilde{P}_{j}$, then $\widetilde{P}_{j} \sim_{s} \widetilde{P}_{i}$;

3. Transitivity: If $\widetilde{P}_{i} \sim_{s} \widetilde{P}_{j}$ and $\widetilde{P}_{j} \sim_{s} \widetilde{P}_{k}$, then $\widetilde{P}_{i} \sim{ }_{s} \widetilde{P}_{k}$

Proof. Let $\widetilde{P}_{i}=c_{1} / s_{B_{1}}+c_{2} / s_{B_{2}}+\cdots+c_{n} / s_{B_{n}}, \widetilde{P}_{j}=$ $d_{1} / s_{B_{1}}+d_{2} / s_{B_{2}}+\cdots+d_{n} / s_{B_{n}}$ and $\widetilde{P}_{k}=e_{1} / s_{B_{1}}+$ $e_{2} / s_{B_{2}}+\cdots+e_{n} / s_{B_{n}}$.

(1) For any fuzzy set $\widetilde{P}_{i}$ on $U$, according to Proposition $1(1), S\left(\widetilde{P}_{i} \ominus \widetilde{P}_{i}\right)=0$, i.e., $\widetilde{P}_{i} \sim_{s} \widetilde{P}_{i}$.

(2) If $\widetilde{P}_{i} \sim_{s} \widetilde{P}_{j}$, then $S\left(\widetilde{P}_{i} \ominus \widetilde{P}_{j}\right)=0$. According to proposition 1 (2), $S\left(\widetilde{P}_{j} \ominus \widetilde{P}_{i}\right)=-S\left(\widetilde{P}_{i} \ominus \widetilde{P}_{j}\right)=0$, hence, $\widetilde{P}_{j} \sim{ }_{s} \widetilde{P}_{i}$.

(3) If $\widetilde{P}_{i} \sim_{s} \widetilde{P}_{j}$ and $\widetilde{P}_{j} \sim{ }_{s} \widetilde{P}_{k}$, then $S\left(\widetilde{P}_{i} \ominus \widetilde{P}_{j}\right)=0$ and $S\left(\widetilde{P}_{j} \ominus \widetilde{P}_{k}\right)=0$. According to Proposition 1 (3), $S\left(\widetilde{P}_{i} \ominus \widetilde{P}_{k}\right)=S\left(\widetilde{P}_{i} \ominus \widetilde{P}_{j}\right)+S\left(\widetilde{P}_{j} \ominus \widetilde{P}_{k}\right)=0$, hence, $\widetilde{P}_{i} \sim{ }_{s} \widetilde{P}_{k}$.

Let $F(U)=\left\{\widetilde{P_{1}}, \widetilde{P_{2}}, \cdots, \widetilde{P_{i}}, \cdots, \widetilde{P_{m}}\right\} \quad(i=$ $1,2, \cdots, m) . \quad \sim_{s}$ is an equivalence relation on fuzzy sets $F(U), F(U) / \sim_{s}$ is the quotient set of $F(U)$ relative to $\sim_{s}$, the equivalence classes of $F(U)$ is denoted by $\left[\widetilde{P}_{i}\right]$, that is, $\forall \widetilde{P}_{i} \in F(U)$, $\left[\widetilde{P}_{i}\right]=\left\{\widetilde{P}_{j} \mid \widetilde{P}_{j} \in F(U) \wedge \widetilde{P}_{i} \sim_{s} \widetilde{P}_{j}\right\}$.

Proposition 5. For any equivalence classes $\left[\widetilde{P}_{i}\right]$ and $\left[\widetilde{P}_{j}\right]$ of $F(U) / \sim_{s}$, if $\widetilde{P}_{i} \leqslant{ }_{s} \widetilde{P}_{j}$, then $\forall \widetilde{P}_{k} \in\left[\widetilde{P}_{i}\right]$ and $\forall \widetilde{P}_{t} \in\left[\widetilde{P}_{j}\right], \widetilde{P}_{k} \leqslant{ }_{s} \widetilde{P}_{t}$.

Proof. If $\widetilde{P}_{i} \leqslant s \widetilde{P}_{j}$, i.e., $S\left(\widetilde{P}_{i} \ominus \widetilde{P}_{j}\right) \leqslant 0 . \forall \widetilde{P}_{k} \in\left[\widetilde{P}_{i}\right]$ and $\forall \widetilde{P}_{t} \in\left[\widetilde{P}_{j}\right]$, we have $S\left(\widetilde{P}_{k} \ominus \widetilde{P}_{i}\right)=S\left(\widetilde{P}_{j} \ominus \widetilde{P}_{t}\right)=0$. According to proposition 1 (3), $S\left(\widetilde{P}_{k} \ominus \widetilde{P}_{t}\right)=S\left(\widetilde{P}_{k} \ominus\right.$ $\left.\widetilde{P}_{i}\right)+S\left(\widetilde{P}_{i} \ominus \widetilde{P}_{t}\right)=S\left(\widetilde{P}_{i} \ominus \widetilde{P}_{t}\right)=S\left(\widetilde{P}_{i} \ominus \widetilde{P}_{j}\right)+S\left(\widetilde{P}_{j} \ominus \widetilde{P}_{t}\right)$ $=S\left(\widetilde{P}_{i} \ominus \widetilde{P}_{j}\right) \leqslant 0$, i.e., $\widetilde{P}_{k} \leqslant{ }_{s} \widetilde{P}_{t}$.

Definition 4. Assume $\left[\widetilde{P}_{1}\right],\left[\widetilde{P}_{2}\right], \cdots,\left[\widetilde{P}_{i}\right], \cdots,\left[\widetilde{P_{m}}\right]$ be $m$ equivalence classes of $F(U) / \sim_{s}$. For any equivalence classes $\left[\widetilde{P}_{i}\right]$ and $\left[\widetilde{P}_{j}\right]$ of $F(U) / \sim_{s},\left[\widetilde{P}_{i}\right] \leqslant \sim_{s}\left[\widetilde{P}_{j}\right]$ if and only if $\widetilde{P}_{i} \leqslant{ }_{s} \widetilde{P}_{j}$.

According to Proposition $5,\left[\widetilde{P}_{i}\right] \leqslant \sim s\left[\widetilde{P}_{j}\right]$ is independent of the choice of the element $\widetilde{P}_{i}$ in $\left[\widetilde{P}_{i}\right]$ and $\widetilde{P}_{j}$ in $\left[\widetilde{P}_{j}\right]$, hence, Definition 4 is well defined. The following properties show that " $\leqslant \sim s$ " is an partially ordered relation on $F(U) / \sim_{s}$.

Proposition 6. For any equivalence classes $\left[\widetilde{P}_{i}\right],\left[\widetilde{P}_{j}\right]$ and $\left[\widetilde{P}_{k}\right]$ of $F(U) / \sim_{s}, " \leqslant \sim s$ " is an partially ordered relation on equivalence classes, i.e., " $\leqslant \sim$ s " satisfies

1. Reflexive: $\left[\widetilde{P}_{i}\right] \leqslant \sim s\left[\widetilde{P}_{i}\right]$;

2. Anti-symmetry:If $\left[\widetilde{P}_{i}\right] \leqslant \sim s\left[\widetilde{P}_{j}\right]$ and $\left[\widetilde{P}_{j}\right] \leqslant \sim s$ $\left[\widetilde{P}_{i}\right]$, then $\left[\widetilde{P}_{i}\right]=\left[\widetilde{P}_{j}\right]$;

3. Transitivity: If $\left[\widetilde{P}_{i}\right] \leqslant \sim s\left[\widetilde{P}_{j}\right],\left[\widetilde{P}_{j}\right] \leqslant \sim s\left[\widetilde{P}_{k}\right]$, then $\left[\widetilde{P}_{i}\right] \leqslant \sim s\left[\widetilde{P}_{k}\right]$.

Proof. (1) For any fuzzy set $\widetilde{P}_{i}$ on $U$, according to Proposition 4 (1), $\widetilde{P}_{i} \leqslant{ }_{s} \widetilde{P}_{i}$, i.e., $\left[\widetilde{P}_{i}\right] \leqslant \sim_{s}\left[\widetilde{P}_{i}\right]$.

(2) If $\left[\widetilde{P}_{i}\right] \leqslant \sim s\left[\widetilde{P}_{j}\right]$, then $\widetilde{P}_{i} \leqslant{ }_{s} \widetilde{P}_{j}$, i.e., $S\left(\widetilde{P}_{i} \ominus \widetilde{P}_{j}\right) \leqslant$ 0 . If $\left[\widetilde{P}_{j}\right] \leqslant \sim s\left[\widetilde{P}_{i}\right]$, then $\widetilde{P}_{j} \leqslant s \widetilde{P}_{i}$, i.e., $S\left(\widetilde{P}_{j} \ominus \widetilde{P}_{i}\right) \leqslant 0$. According to proposition $1, S\left(\widetilde{P}_{i} \ominus \widetilde{P}_{j}\right)=-S\left(\widetilde{P}_{j} \ominus\right.$ $\left.\widetilde{P}_{i}\right)$, i.e, $S\left(\widetilde{P}_{i} \ominus \widetilde{P}_{j}\right)=0$. Since $\widetilde{P}_{i} \sim_{s} \widetilde{P}_{j}$, i.e., $\left[\widetilde{P}_{i}\right]=\left[\widetilde{P}_{j}\right]$.

(3) If $\left[\widetilde{P}_{i}\right] \leqslant \sim s\left[\widetilde{P}_{j}\right]$ and $\left[\widetilde{P}_{j}\right] \leqslant \sim s\left[\widetilde{P}_{k}\right]$, then $\widetilde{P}_{i} \leqslant{ }_{s} \widetilde{P}_{j}$ and $\widetilde{P}_{j} \leqslant s \widetilde{P}_{k}$. According to proposition $2(3), \widetilde{P}_{i} \leqslant s$ $\widetilde{P}_{k}$, hence, $\left[\widetilde{P}_{i}\right] \leqslant \sim s\left[\widetilde{P}_{k}\right]$.

Furthermore, for any equivalence classes $\left[\widetilde{P}_{i}\right]$ and $\left[\widetilde{P}_{j}\right]$ of $F(U) / \sim_{s}$, we have $\left[\widetilde{P}_{i}\right] \leqslant \sim_{s}\left[\widetilde{P}_{j}\right]$ or $\left[\widetilde{P}_{j}\right] \leqslant \sim_{s}$ $\left[\widetilde{P}_{i}\right]$ according to Proposition 3 and Definition 4 . Therefore, " $\leqslant \sim s$ " is a linear ordering relation on $F(U) / \sim_{s}$.

Example 4. Let $\widetilde{P}_{1}, \quad \widetilde{P}_{2}, \quad \widetilde{P}_{3}, \quad \widetilde{P}_{4}$ and $\widetilde{P}_{5}$ be five scores of alternative $x_{j}(1 \leqslant j \leqslant 5)$, which are fuzzy sets on $U=\left\{s_{-4}, s_{-3}, \cdots, s_{4}\right\}$, where $\widetilde{P}_{1}=0.045 / s_{-1}+0.165 / s_{0}+0.4 / s_{1}+0.25 / s_{2}+$ $0.14 / s_{3}, \quad \widetilde{P_{2}}=0.14 / s_{0}+0.17 / s_{1}+0.315 / s_{2}+$ $0.285 / s_{3}+0.09 / s_{4}, \widetilde{P}_{3}=0.005 / s_{-3}+0.06 / s_{-2}+$ $0.105 / s_{-1}+0.28 / s_{0}+0.85 / s_{1}+0.11 / s_{2}+0.06 / s_{3}$, $\widetilde{P}_{4}=0.08 / s_{-4}+0.105 / s_{-3}+0.145 / s_{-1}+0.16 / s_{0}+$ $0.18 / s_{1}+0.11 / s_{2}+0.31 / s_{3}+0.115 / s_{4}$ and $\widetilde{P}_{5}=$ $0.08 / s_{-3}+0.1 / s_{-1}+0.08 / s_{0}+0.06 / s_{1}+0.26 / s_{2}+$ $0.385 / s_{3}+0.155 / s_{4}$.

According to (1), we have $S\left(\widetilde{P_{1}} \ominus \widetilde{P_{2}}\right)=$ $S\left(\left(0.045 / s_{-1}+0.165 / s_{0}+0.4 / s_{1}+0.25 / s_{2}+\right.\right.$ $\left.0.14 / s_{3}\right) \ominus\left(0.14 / s_{0}+0.17 / s_{1}+0.315 / s_{2}+\right.$ $\left.\left.0.285 / s_{3}+0.09 / s_{4}\right)\right)=(-1) \times(0.045-0)+0 \times$ 
$(0.165-0.14)+1 \times(0.4-0.17)+2 \times(0.25-$ $0.315)+3 \times(0.14-0.285)+4 \times(0-0.09)=$ -0.74. Similarly, $S\left(\widetilde{P}_{1} \ominus \widetilde{P}_{3}\right)=0.265, S\left(\widetilde{P}_{1} \ominus \widetilde{P}_{4}\right)=$ $0.265, S\left(\widetilde{P}_{1} \ominus \widetilde{P}_{5}\right)=-0.74, S\left(\widetilde{P}_{2} \ominus \widetilde{P}_{3}\right)=1.005$, $S\left(\widetilde{P}_{2} \ominus \widetilde{P}_{4}\right)=1.005, S\left(\widetilde{P}_{2} \ominus \widetilde{P}_{5}\right)=0, S\left(\widetilde{P}_{2} \ominus \widetilde{P}_{5}\right)=$ $0, \quad S\left(\widetilde{P}_{3} \ominus \widetilde{P}_{4}\right)=0, \quad S\left(\widetilde{P}_{3} \ominus \widetilde{P}_{5}\right)=-0.1005$ and $S\left(\widetilde{P}_{4} \ominus \widetilde{P}_{5}\right)=-0.1005$.

Accordingly, we get three equivalence class of $F(U) / \sim_{s}$, i.e., $\left[\widetilde{P}_{1}\right]=\left\{\widetilde{P}_{1}\right\},\left[\widetilde{P}_{2}\right]=\left\{\widetilde{P}_{2}, \widetilde{P}_{5}\right\},\left[\widetilde{P}_{3}\right]=$ $\left\{\widetilde{P}_{3}, \widetilde{P}_{4}\right\}$. Based on $S\left(\widetilde{P}_{1} \ominus \widetilde{P}_{2}\right)=-0.74, S\left(\widetilde{P}_{1} \ominus \widetilde{P}_{3}\right)=$ 0.265 and $S\left(\widetilde{P}_{2} \ominus \widetilde{P}_{3}\right)=1.005$, we have

$$
\left[\widetilde{P}_{3}\right] \leqslant \sim s\left[\widetilde{P}_{1}\right] \leqslant \sim s\left[\widetilde{P}_{2}\right] .
$$

The following Proposition 7 shows that the equivalence classes of $F(U) / \sim_{s}$ is closed for weighted aggregation operator.

Proposition 7. For any equivalence class $\left[\widetilde{P}_{i}\right]$ of $F(U) / \sim_{s}$, if $\widetilde{P_{i 1}}, \cdots, \widetilde{P_{i m}} \in\left[\widetilde{P}_{i}\right]$, then $\left(w_{1} \otimes \widetilde{P_{i 1}}\right) \oplus$ $\cdots \oplus\left(w_{m} \otimes \widetilde{P_{i m}}\right) \in\left[\widetilde{P}_{i}\right]$, where $w_{i} \in[0,1]$ and $\sum_{i=1}^{m} w_{i}=1,(i=1, \cdots, m)$.

Proof. Let $\widetilde{P_{i 1}}=a_{1} / s_{B_{1}}+a_{2} / s_{B_{2}}+\cdots+a_{n} / s_{B_{n}}$, $\widetilde{P_{i 2}}=b_{1} / s_{B_{1}}+b_{2} / s_{B_{2}}+\cdots+b_{n} / s_{B_{n}}, \cdots, \widetilde{P_{i m}}=$ $l_{1} / s_{B_{1}}+l_{2} / s_{B_{2}}+\cdots+l_{n} / s_{B_{n}}$ and $\widetilde{P}_{t}=\left(w_{1} \otimes \widetilde{P_{i 1}}\right) \oplus$ $\cdots \oplus\left(w_{n} \otimes \widetilde{P_{i m}}\right)=\left[w_{1} a_{1}+w_{2} b_{1}+\cdots+w_{n} l_{1}\right] / S_{B_{1}}+$ $\left[w_{1} a_{2}+w_{2} b_{2}+\cdots+w_{m} l_{2}\right] / S_{B_{2}}+\cdots+\left[w_{1} a_{n}+\right.$ $\left.w_{2} b_{n}+\cdots+w_{n} l_{n}\right] / S_{B_{1}}$. If $\widetilde{P_{i 1}}, \cdots, \widetilde{P_{i m}} \in\left[\widetilde{P}_{i}\right]$, according to Definition 3, $\forall \widetilde{P_{i j}}, \widetilde{P_{i k}} \in\left[\widetilde{P}_{i}\right](j, k=$ $1,2, \cdots, m)$, we have $S\left(\widetilde{P_{i j}} \ominus \widetilde{P_{i k}}\right)=0$. Since, $S\left(\widetilde{P_{t}} \ominus \widetilde{P_{i 1}}\right)=B_{1} \times\left[\left(w_{1}-1\right) a_{1}+w_{2} b_{1}+w_{3} c_{1}+\right.$ $\left.\cdots+w_{m} l_{1}\right]+B_{2} \times\left[\left(w_{1}-1\right) a_{2}+w_{2} b_{2}+w_{3} c_{2}+\right.$ $\left.\cdots+w_{m} l_{2}\right]+\cdots+B_{n} \times\left[\left(w_{1}-1\right) a_{n}+w_{2} b_{n}+w_{3} c_{n}+\right.$ $\left.\cdots+w_{m} l_{n}\right]=B_{1} \times\left[\left(-w_{2}-w_{3}-\cdots-w_{m}\right) a_{1}+\right.$ $\left.w_{2} b_{1}+w_{3} c_{1}+\cdots+w_{m} l_{1}\right]+B_{2} \times\left[\left(-w_{2}-w_{3}-\right.\right.$ $\left.\left.\cdots-w_{m}\right) a_{2}+w_{2} b_{2}+w_{3} c_{2}+\cdots+w_{m} l_{2}\right]+\cdots+B_{n} \times$ $\left[\left(-w_{2}-w_{3}-\cdots-w_{m}\right) a_{n}+w_{2} b_{n}+w_{3} c_{n}+\cdots+\right.$ $\left.w_{m} l_{n}\right]=B_{1} \times\left[w_{2}\left(b_{1}-a_{1}\right)+w_{3}\left(c_{1}-a_{1}\right)+\cdots+\right.$ $\left.w_{m}\left(l_{1}-a_{1}\right)\right]+B_{2} \times\left[w_{2}\left(b_{2}-a_{2}\right)+w_{3}\left(c_{2}-a_{2}\right)+\right.$ $\left.\cdots+w_{m}\left(l_{2}-a_{2}\right)\right]+\cdots+B_{n} \times\left[w_{2}\left(b_{n}-a_{n}\right)+w_{3}\left(c_{n}-\right.\right.$ $\left.\left.a_{n}\right)+\cdots+w_{m}\left(l_{n}-a_{n}\right)\right]=w_{2}\left[B_{1}\left(b_{1}-a_{1}\right)+B_{2}\left(b_{2}-\right.\right.$ $\left.a_{2}\right)+\cdots+B_{n}\left(b_{n}-a_{n}\right)+w_{3}\left[B_{1}\left(c_{1}-a_{1}\right)+B_{2}\left(c_{2}-\right.\right.$ $\left.a_{2}\right)+\cdots+B_{n}\left(c_{n}-a_{n}\right)+\cdots+w_{m}\left[B_{1}\left(l_{1}-a_{1}\right)+\right.$ $B_{2}\left(l_{2}-a_{2}\right)+\cdots+B_{n}\left(l_{n}-a_{n}\right)=w_{2} S\left(\widetilde{P_{i 2}} \ominus \widetilde{P_{i 1}}\right)+$ $w_{3} S\left(\widetilde{P_{i 3}} \ominus \widetilde{P_{i 1}}\right)+\cdots+w_{m} S\left(\widetilde{P_{i m}} \ominus \widetilde{P_{i 1}}\right)=0$, i.e., $\left(w_{1} \otimes\right.$ $\left.\widetilde{P_{i 1}}\right) \oplus \cdots \oplus\left(w_{n} \otimes \widetilde{P_{i m}}\right) \in\left[\widetilde{P}_{i}\right]$.

In practical decision-making, we always find more than one alternatives have the same effect, such as in Example 4, $\left[\widetilde{P}_{2}\right]=\left\{\widetilde{P}_{2}, \widetilde{P}_{5}\right\}$ is maximum equivalence class. According to Proposition 7, we adopt the following method to understand why $\left\{x_{2}, x_{5}\right\}$ is the better choose, assume that the weighting vector $W=\left[w_{1}, w_{2}\right]^{T}=[0.3,0.7]^{T}$, according to Proposition 7, $\widetilde{P}_{6}=\left(w_{1} \otimes \widetilde{P}_{2}\right) \oplus\left(w_{2} \otimes \widetilde{P}_{5}\right)=$ $\left[0.3 \otimes\left(0.14 / s_{0}+0.17 / s_{1}+0.315 / s_{2}+0.285 / s_{3}+\right.\right.$ $\left.\left.0.09 / s_{4}\right)\right] \oplus\left[0.7 \otimes\left(0.08 / s_{-3}+0.1 / s_{-1}+0.08 / s_{0}+\right.\right.$ $\left.\left.0.06 / s_{1}+0.26 / s_{2}+0.385 / s_{3}+0.155 / s_{4}\right)\right]=$ $0.056 / s_{-3}+0.07 / s_{-1}+0.098 / s_{0}+0.093 / s_{1}+$ $0.2765 / s_{2}+0.355 / s_{3}+0.1355 / s_{4} . \quad \widetilde{P}_{6}$ is the final evaluation value about $x_{2}$ and $x_{5}$.

\subsection{An improve method of fuzzy group decision-making}

Assume that there are $n$ alternatives $\left\{x_{1}, x_{2}, \cdots, x_{n}\right\}$, $m$ attributes $\left\{f_{1}, f_{2}, \cdots, f_{m}\right\}$ and $g$ decision-makers $\left\{D_{1}, D_{2}, \cdots, D_{g}\right\}$. Let $H=\left[h_{1}, h_{2}, \cdots, h_{g}\right]^{T}$ be the weighting vector of the decision-makers, where $h_{i}$ denotes the weight of decision-maker $D_{i}, 1 \leqslant i \leqslant g$ and $\sum_{i=1}^{g} h_{i}=1$. Let $V=\left[v_{1}, v_{2}, \cdots, v_{m}\right]^{T}$ be the weighting vector of the attributes, where $v_{i}$ denotes the weight of attribute $f_{i}, 1 \leqslant i \leqslant m$ and $\sum_{i=1}^{m} v_{i}=1$. The proposed algorithm for fuzzy group decisionmaking is presented as follows:

Step1: Construct the fuzzy evaluating matrix $\widetilde{F}_{k}$ for decision- maker $D_{k}$ with respect to attribute $f_{i}$ of the alternative $x_{j}$,

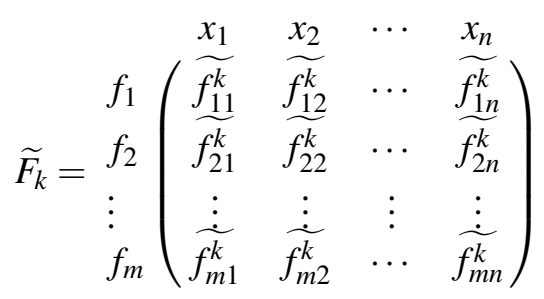

Step2: Assume that the weighting vector $W=\left[w_{1}\right.$, $\left.w_{2}, \cdots, w_{g}\right]^{T}$. Based on weighting vector $W$ and the fuzzy evaluating matrix $\widetilde{F_{k}}$, the weight $\widetilde{Z_{i j}}$ of the attribute $f_{i}$ of the alternative $x_{j}$ is calculated by the 
FIOWA operators, i.e.,

$$
\begin{aligned}
\widetilde{Z_{i j}} & =F_{\text {FIOWA }}\left(\left\langle h_{1}, \widetilde{f_{i j}^{1}}\right\rangle, \cdots,\left\langle h_{k}, \widetilde{f_{i j}^{k}}\right\rangle\right) \\
& =w_{1} \widetilde{f_{i j}^{\prime}} \oplus \cdots \oplus w_{k} \widetilde{f_{i j}^{\prime k}}
\end{aligned}
$$

where, $1 \leqslant i \leqslant m, 1 \leqslant j \leqslant n, 1 \leqslant k \leqslant g, h_{k}$ denotes the weighting vector of decision-maker $D_{k}, \widetilde{f_{i j}^{\prime k}}$ is the value of the OWA pair having the $k$ th largest order inducing $h$ value.

Step3: Assume that the weighting vector $R=$ $\left[r_{1}, r_{2}, \cdots, r_{i}\right]^{T}$. Based on weighting vector $R$ and the weighted value $\widetilde{Z_{i j}}$, the score $\widetilde{E_{j}}$ of alternative $x_{j}$ is calculated as follows:

$$
\begin{aligned}
\widetilde{E_{j}} & =F_{\text {FIOWA }}\left(\left\langle v_{1}, \widetilde{Z_{1 j}}\right\rangle, \cdots,\left\langle v_{i}, \widetilde{Z_{i j}}\right\rangle\right) \\
& =r_{1} \widetilde{Z_{1 j}} \oplus \cdots \oplus r_{i}{\widetilde{Z_{i j}}}^{\prime}
\end{aligned}
$$

where $1 \leqslant i \leqslant m, 1 \leqslant j \leqslant n, v_{i}$ denotes the weight of attribute $f_{i}, \widetilde{Z_{i j}}$ is the value of the OWA pair having the $k$ th largest order inducing $v$ value.

step4: Based on (1), the score $S\left(\widetilde{E}_{i} \ominus \widetilde{E}_{j}\right)$ of the weighted difference of membership values between $\widetilde{E}_{i}$ and $\widetilde{E}_{j}$ is calculated, where $1 \leqslant j \leqslant n$.

step5: All evaluations of alternatives are classified by the equivalence relation $\sim_{s}$.

step6: The order of sort $\left\{\left[\widetilde{E_{1}}\right],\left[\widetilde{E_{2}}\right], \cdots,\left[\widetilde{E_{m}}\right]\right\}$ can be obtained by Definition 4 .

step7: Assume $\left[\widetilde{E_{i}}\right]=\max \left\{\left[\widetilde{E_{1}}\right],\left[\widetilde{E_{2}}\right], \cdots,\left[\widetilde{E_{m}}\right]\right\}$. If $\left|\left[\widetilde{E}_{i}\right]\right|=1$, then the better choose of the alternative is $x_{i}$. Else $\left|\left[\widetilde{E}_{i}\right]\right|>1$, we used weighted average operator to aggregation element of equivalence class $\left[\widetilde{E}_{i}\right]$. The result of aggregation $\widetilde{E}_{j} \in\left[\widetilde{E}_{i}\right]$ is the final evaluation value of better alternatives.

\section{Numerical examples}

In this section, we use two examples to illustrate the proposed method for handling fuzzy group decisionmaking problems.

Example 5. Assume that there are five alternative $x_{1}, x_{2}, x_{3}, x_{4}$ and $x_{5}$ and three decision-makers $D_{1}$, $D_{2}$ and $D_{3}$ who want to choose the best alternative among $x_{1}, x_{2}, x_{3}, x_{4}$ and $x_{5}$. Assume that there are four attributes, i.e., the risk analysis (denoted by $f_{1}$ ), the growth analysis (denoted by $f_{2}$ ), the socialpolitical impact analysis (denoted by $f_{3}$ ) and the environmental impact analysis (denoted by $f_{4}$ ). Assume that the weighting vector $H$ of the decisionmakers is shown as follows: $H=\left[h_{1}, h_{2}, h_{3}\right]^{T}=$ $[0.2,0.5,0.3]^{T}$, where $h_{i}$ denotes the weight of the decision-maker $D_{i}$ and $1 \leqslant i \leqslant 3$. Assume that weighting vector $V$ of the four attributes is shown as follows: $V=\left[v_{1}, v_{2}, v_{3}, v_{4}\right]^{T}=[0.3,0.4,0.2,0.1]^{T}$, where $v_{i}$ denotes the weight of the attribute $f_{i}$ and $1 \leqslant i \leqslant 4$. Assume that there are nine linguistic terms $s_{-4}, s_{-3}, s_{-2}, s_{-1}, s_{0}, s_{1}, s_{2}, s_{3}$ and $s_{4}$, where $s_{-4}=$ extremely poor, $s_{-3}=$ very poor, $s_{-2}=$ poor, $s_{-1}=$ slightly poor, $s_{0}=$ fair, $s_{1}=$ slightly good, $s_{2}=$ good, $s_{3}=$ very good, $s_{4}=$ extremely good. Assume that the fuzzy evaluating values of the alternatives given by the decision-makers with respect to different attributes as following:

Step1: Construct the fuzzy evaluating matrix $\widetilde{F}_{k}$ for decision- maker $D_{k}$ with respect to attribute $f_{i}$ of the alternative $x_{j}$, where $1 \leqslant k \leqslant 3,1 \leqslant i \leqslant 4$, $1 \leqslant j \leqslant 5$ shown as follows:

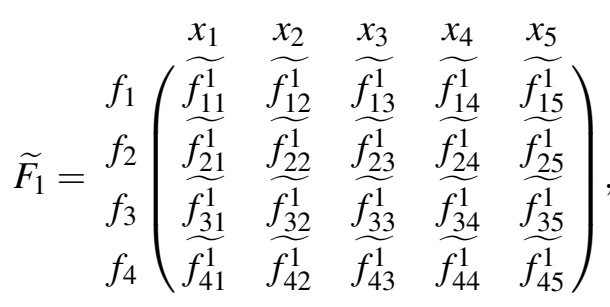

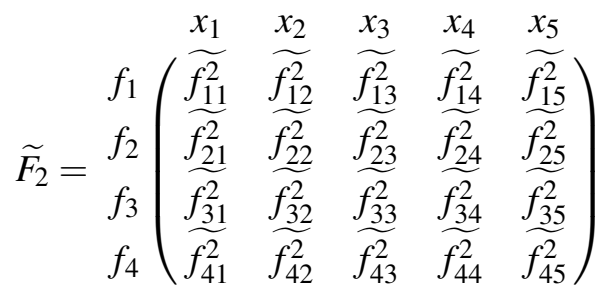

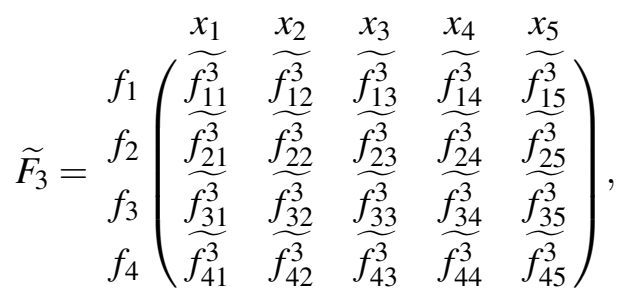


where $\widetilde{f_{11}^{1}}=0.1 / s_{-1}+0.5 / s_{0}, \widetilde{f_{12}^{1}}=0.5 / s_{0}+$ $0.4 / s_{2}, \widetilde{f_{13}^{1}}=0.05 / s_{-3}+0.3 / s_{0}+0.9 / s_{1}+0.45 / s_{2}$, $\widetilde{f_{14}^{1}}=0.6 / s_{0}+0.5 / s_{3}+0.1 / s_{4}, \widetilde{f_{15}^{1}}=0.85 / s_{2}+$ $0.9 / s_{3}, \widetilde{f_{21}^{1}}=0.5 / s_{0}+0.7 / s_{1}, \widetilde{f_{22}^{1}}=0.7 / s_{3}+0.5 / s_{4}$, $\widetilde{f_{23}^{1}}=0.4 / s_{0}+0.7 / s_{1}, \widetilde{f_{24}^{1}}=0.1 / s_{-4}+0.32 / s_{-1}+$ $0.15 / s_{3}, \widetilde{f_{25}^{1}}=0.5 / s_{-3}+0.2 / s_{3}, \widetilde{f_{31}^{1}}=0.1 / s_{1}+$ $0.9 / s_{2}, \widetilde{f_{32}^{1}}=0.3 / s_{2}+0.2 / s_{4}, \widetilde{f_{33}^{1}}=0.05 / s_{-3}+$ $0.5 / s_{-1}+0.6 / s_{0}+0.9 / s_{1}, \widetilde{f_{34}^{1}}=0.5 / s_{1}+0.1 / s_{2}+$ $0.9 / s_{3}+0.85 / s_{4}, \widetilde{f_{35}^{1}}=0.5 / s_{-1}+0.3 / s_{1}+0.1 / s_{3}$, $\widetilde{f_{41}^{1}}=0.9 / s_{0}+0.6 / s_{2}, \quad \widetilde{f_{42}^{1}}=0.6 / s_{2}+0.15 / s_{4}$, $\widetilde{f_{43}^{1}}=0.5 / s_{-2}+0.5 / s_{1}, \widetilde{f_{44}^{1}}=0.5 / s_{0}+0.5 / s_{1}+$ $0.5 / s_{2}+0.85 / s_{3}, \widetilde{f_{45}^{1}}=0.8 / s_{2}+0.7 / s_{3}+0.1 / s_{4}$, $\widetilde{f_{11}^{2}}=0.1 / s_{0}+0.7 / s_{1}, \quad \widetilde{f_{12}^{2}}=0.6 / s_{0}+0.4 / s_{2}$, $\widetilde{f_{13}^{2}}=0.1 / s_{0}+0.9 / s_{1}+0.4 / s_{3}, \widetilde{f_{14}^{2}}=0.5 / s_{-3}+$ $0.5 / s_{-1}, \widetilde{f_{15}^{2}}=0.8 / s_{2}+0.9 / s_{3}+0.6 / s_{4}, \widetilde{f_{21}^{2}}=$ $0.8 / s_{1}+0.3 / s_{3}, \widetilde{f_{22}^{2}}=0.3 / s_{1}+0.8 / s_{3}, \quad \widetilde{f_{23}^{2}}=$ $0.11 / s_{-2}+0.84 / s_{1}, \widetilde{f_{24}^{2}}=0.2 / s_{0}+0.3 / s_{1}+0.1 / s_{3}$, $\widetilde{f_{25}^{2}}=0.2 / s_{-3}+0.11 / s_{0}, \widetilde{f_{31}^{2}}=0.9 / s_{2}+0.11 / s_{3}$, $\widetilde{f_{32}^{2}}=0.2 / s_{0}+0.4 / s_{2}, \widetilde{f_{33}^{2}}=0.5 / s_{-1}+0.6 / s_{0}+$ $0.9 / s_{1}+0.2 / s_{2}, \widetilde{f_{34}^{2}}=0.1 / s_{0}+0.5 / s_{1}+0.6 / s_{2}+$ $0.9 / s_{3}+0.6 / s_{4}, \widetilde{f_{35}^{2}}=0.8 / s_{-1}+0.4 / s_{0}, \widetilde{f_{41}^{2}}=$ $0.3 / s_{-1}+0.7 / s_{0}+0.68 / s_{2}, \widetilde{f_{42}^{2}}=0.7 / s_{1}+0.7 / s_{2}$, $\widetilde{f_{43}^{2}}=0.2 / s_{-2}+0.1 / s_{-1}+0.1 / s_{0}+0.86 / s_{1}, \widetilde{f_{44}^{2}}=$ $0.3 / s_{-3}+0.3 / s_{-1}+0.2 / s_{0}+0.2 / s_{1}+0.84 / s_{3}$, $\widetilde{f_{45}^{2}}=0.8 / s_{2}+0.3 / s_{3} \widetilde{f_{11}^{3}}=0.1 / s_{-1}+0.5 / s_{1}, \widetilde{f_{12}^{3}}=$ $0.9 / s_{2}+0.5 / s_{3}, \widetilde{f_{13}^{3}}=0.9 / s_{1}+0.3 / s_{0}+0.7 / s_{2}$, $\widetilde{f_{14}^{3}}=0.6 / s_{0}+0.1 / s_{4}, \widetilde{f_{15}^{3}}=0.1 / s_{2}+0.9 / s_{3}+$ $0.7 / s_{4}, \widetilde{f_{21}^{3}}=0.2 / s_{1}+0.5 / s_{3}, \widetilde{f_{22}^{3}}=0.5 / s_{1}+$ $0.2 / s_{3}, \widetilde{f_{23}^{3}}=0.15 / s_{-2}+0.4 / s_{0}+0.8 / s_{1}, \widetilde{f_{24}^{3}}=$ $0.6 / s_{-4}+0.12 / s_{-1}, \widetilde{f_{25}^{3}}=0.15 / s_{0}+0.2 / s_{3}, \widetilde{f_{31}^{3}}=$ $0.1 / s_{1}+0.9 / s_{2}+0.15 / s_{3}, \widetilde{f_{32}^{3}}=0.8 / s_{2}+0.2 / s_{4}$, $\widetilde{f_{33}^{3}}=0.5 / s_{-1}+0.6 / s_{0}+0.9 / s_{1}, \widetilde{f_{34}^{3}}=0.5 / s_{1}+$ $0.6 / s_{2}+0.9 / s_{3}+0.1 / s_{4}, \widetilde{f_{35}^{3}}=0.8 / s_{1}+0.6 / s_{3}$, $\widetilde{f_{41}^{3}}=0.5 / s_{-1}+0.9 / s_{0}+0.8 / s_{2}, \widetilde{f_{42}^{3}}=0.5 / s_{1}+$ $0.1 / s_{2}, \widetilde{f_{43}^{3}}=0.5 / s_{0}+0.9 / s_{1}, \widetilde{f_{44}^{3}}=0.5 / s_{-3}+$
$0.5 / s_{-1}+0.3 / s_{3}, \widetilde{f_{45}^{3}}=0.8 / s_{2}+0.2 / s_{3}$.

Step2: Assume that the weighting vector $W=$ $\left[w_{1}, w_{2}, w_{3}\right]^{T}=[0.5,0.3,0.2]^{T}$. The weight $\widetilde{Z_{i j}}$ of the attribute $f_{i}$ of the alternative $x_{j}$ is calculated by the FIOWA operators, i.e.,

$$
\begin{aligned}
\widetilde{Z_{i j}} & =F_{F I O W A}\left(\left\langle h_{1}, \widetilde{f_{i j}^{1}}\right\rangle,\left\langle h_{2}, \widetilde{f_{i j}^{2}}\right\rangle,\left\langle h_{3}, \widetilde{f_{i j}^{3}}\right\rangle\right) \\
& =w_{1} \widetilde{f_{i j}^{\prime} 1} \oplus w_{2} \widetilde{f_{i j}^{\prime \prime}} \oplus w_{3} \tilde{f}_{i j}^{\prime 3},
\end{aligned}
$$

where $h_{k}$ denotes the weighting vector of decisionmakers $D_{k}, 1 \leqslant i \leqslant 4,1 \leqslant j \leqslant 5,1 \leqslant k \leqslant 3$. $\widetilde{f_{i j}^{\prime} k}$ is the value of the OWA pair having the $k$ th largest order inducing $h$ value. The results are shown as follows: $\widetilde{Z_{11}}=0.05 / s_{-1}+0.15 / s_{0}+0.5 / s_{1}, \widetilde{Z_{21}}=0.1 / s_{0}+$ $0.6 / s_{1}+0.3 / s_{3}, \widetilde{Z_{31}}=0.05 / s_{1}+0.9 / s_{2}+0.1 / s_{3}$, $\widetilde{Z_{41}}=0.3 / s_{-1}+0.8 / s_{0}+0.7 / s_{2}, \widetilde{Z_{12}}=0.4 / s_{0}+$ $0.55 / s_{2}+0.15 / s_{3}, \widetilde{Z_{22}}=0.3 / s_{1}+0.6 / s_{3}+0.1 / s_{4}$, $\widetilde{Z_{32}}=0.1 / s_{0}+0.5 / s_{2}+0.1 / s_{4}, \widetilde{Z_{42}}=0.5 / s_{1}+$ $0.5 / s_{2}+0.3 / s_{4}, \widetilde{Z_{13}}=0.01 / s_{-3}+0.2 / s_{0}+0.9 / s_{1}+$ $0.3 / s_{2}+0.2 / s_{3}, \widetilde{Z_{23}}=0.1 / s_{-2}+0.2 / s_{0}+0.8 / s_{1}$, $\widetilde{Z_{33}}=0.01 / s_{-3}+0.5 / s_{-1}+0.6 / s_{0}+0.9 / s_{1}+$ $0.1 / s_{2}, \widetilde{Z_{43}}=0.2 / s_{-2}+0.05 / s_{-1}+0.2 / s_{0}+0.8 / s_{1}$, $\widetilde{Z_{14}}=0.25 / s_{-3}+0.25 / s_{-1}+0.3 / s_{0}+0.1 / s_{3}+$ $0.05 / s_{4}, \quad \widetilde{Z_{24}}=0.2 / s_{-4}+0.1 / s_{-1}+0.1 / s_{0}+$ $0.15 / s_{1}+0.08 / s_{3}, \quad \widetilde{Z_{34}}=0.05 / s_{0}+0.5 / s_{1}+$ $0.5 / s_{2}+0.9 / s_{3}+0.5 / s_{4}, \widetilde{Z_{44}}=0.3 / s_{-3}+0.3 / s_{-1}+$ $0.2 / s_{0}+0.2 / s_{2}+0.1 / s_{2}+0.68 / s_{3}, \widetilde{Z_{15}}=0.6 / s_{2}+$ $0.9 / s_{3}+0.51 / s_{4}, \widetilde{Z_{25}}=0.2 / s_{-3}+0.1 / s_{0}+0.1 / s_{3}$, $\widetilde{Z_{35}}=0.5 / s_{-1}+0.2 / s_{0}+0.3 / s_{1}+0.2 / s_{3}, \widetilde{Z_{45}}=$ $0.8 / s_{2}+0.35 / s_{3}+0.02 / s_{4}$.

Step3: Assume that the weighting vector $R=$ $\left[r_{1}, r_{2}, r_{3}, r_{4}\right]^{T}=[0.4,0.3,0.2,0.1]^{T}$, we get the evaluations value $\widetilde{E}_{j}$ of alternative $x_{j}$ by using FIOWA operator, i.e.,

$$
\begin{aligned}
\widetilde{E_{j}} & =F_{\text {FIOWA }}\left(\left\langle v_{1}, \widetilde{Z_{1 j}}\right\rangle,\left\langle v_{2}, \widetilde{Z_{2 j}}\right\rangle,\left\langle v_{3}, \widetilde{Z_{3 j}}\right\rangle,\left\langle v_{4}, \widetilde{Z_{4 j}}\right\rangle\right) \\
& =r_{1}{\widetilde{Z_{1 j}}}^{\prime} \oplus{\widetilde{r_{2}}}_{Z_{2 j}}^{\prime} \oplus{\widetilde{r_{3}}}_{Z_{3 j}}^{\prime} \oplus{\widetilde{r_{4}}}_{Z_{4 j}}^{\prime},
\end{aligned}
$$

$v_{i}$ denotes the weight of attribute $f_{i},{\widetilde{Z_{i j}}}^{\prime}$ is the value of the OWA pair having the $i$ th largest order inducing $v$ value, $1 \leqslant i \leqslant 4$, $1 \leqslant j \leqslant 5$. The results are shown by $\widehat{E_{1}}=$ $F_{\text {FIOWA }}\left(\left\langle v_{1}, \widetilde{Z_{11}}\right\rangle,\left\langle v_{2}, \widetilde{Z_{21}}\right\rangle,\left\langle v_{3}, \widetilde{Z_{31}}\right\rangle,\left\langle v_{4}, \widetilde{Z_{41}}\right\rangle\right)=$ $r_{1}{\widetilde{Z_{11}}}^{\prime} \oplus r_{2}{\widetilde{Z_{21}}}^{\prime} \oplus r_{3} \widetilde{Z_{31}} \oplus r_{4}{\widetilde{Z_{41}}}^{\prime}=0.4 \otimes\left(0.1 / s_{0}+\right.$ 
$\left.0.6 / s_{1}+0.3 / s_{3}\right) \oplus 0.3 \otimes\left(0.05 / s_{-1}+0.15 / s_{0}+\right.$ $\left.0.5 / s_{1}\right) \oplus 0.2 \otimes\left(0.05 / s_{1}+0.9 / s_{2}+0.1 / s_{3}\right) \oplus$ $0.1 \otimes\left(0.3 / s_{-1}+0.8 / s_{0}+0.7 / s_{2}\right)=0.045 / s_{-1}+$ $0.165 / s_{0}+0.4 / s_{1}+0.25 / s_{2}+0.14 / s_{3}$. Similarly, $\widetilde{E_{2}}=0.14 / s_{0}+0.17 / s_{1}+0.315 / s_{2}+0.285 / s_{3}+$ $0.09 / s_{4}, \widetilde{E_{3}}=0.005 / s_{-3}+0.06 / s_{-2}+0.105 / s_{-1}+$ $0.28 / s_{0}+0.85 / s_{1}+0.11 / s_{2}+0.06 / s_{3}, \quad \widetilde{E_{4}}=$ $0.08 / s_{-4}+0.105 / s_{-3}+0.145 / s_{-1}+0.16 / s_{0}+$ $0.18 / s_{1}+0.11 / s_{2}+0.31 / s_{3}+0.115 / s_{4}$ and $\widetilde{E_{5}}=$ $0.08 / s_{-3}+0.1 / s_{-1}+0.08 / s_{0}+0.06 / s_{1}+0.26 / s_{2}+$ $0.385 / s_{3}+0.155 / s_{4}$.

Step4: Based on (1), the score $S\left(\widetilde{E}_{i} \ominus \widetilde{E}_{j}\right)$ of the weighted difference of membership values between $\widetilde{E}_{i}$ and $\widetilde{E}_{j}$ is calculated, where $1 \leqslant j \leqslant 5, \quad 1 \leqslant i \leqslant 5$. The results are shown by $S\left(\widetilde{E_{1}} \ominus \widetilde{E_{2}}\right)=S\left(\left(0.045 / s_{-1}+0.165 / s_{0}+\right.\right.$ $\left.0.4 / s_{1}+0.25 / s_{2}+0.14 / s_{3}\right) \ominus\left(0.14 / s_{0}+0.17 / s_{1}+\right.$ $\left.\left.0.315 / s_{2}+0.285 / s_{3}+0.09 / s_{4}\right)\right)=(-1) \times(0.045-$ $0)+0 \times(0.165-0.14)+1 \times(0.4-0.17)+2 \times$ $(0.25-0.315)+3 \times(0.14-0.284)+4 \times(0-$ $0.09)=-0.74$. Similarly, $S\left(\widetilde{E_{1}} \ominus \widetilde{E_{3}}\right)=0.265$, $S\left(\widetilde{E_{1}} \ominus \widetilde{E_{4}}\right)=0.265, S\left(\widetilde{E_{1}} \ominus \widetilde{E_{5}}\right)=-0.74, S\left(\widetilde{E_{2}} \ominus\right.$ $\left.\widetilde{E_{3}}\right)=1.005, S\left(\widetilde{E_{2}} \ominus \widetilde{E_{4}}\right)=1.005, S\left(\widetilde{E_{2}} \ominus \widetilde{E_{5}}\right)=0$. $S\left(\widetilde{E_{3}} \ominus \widetilde{E_{4}}\right)=0, S\left(\widetilde{E_{3}} \ominus \widetilde{E_{5}}\right)=-0.1005$ and $S\left(\widetilde{E_{4}} \ominus\right.$ $\left.\widetilde{E_{5}}\right)=-0.1005$.

Step5: All evaluations of alternatives are classified by the equivalence relation $\sim_{s}$, therefore, we have $\left[\widetilde{E_{1}}\right]=\left\{\widetilde{E_{1}}\right\},\left[\widetilde{E_{2}}\right]=\left\{\widetilde{E_{2}}, \widetilde{E_{5}}\right\},\left[\widetilde{E_{3}}\right]=\left\{\widetilde{E_{3}}, \widetilde{E_{4}}\right\}$.

Step6: Sort the equivalence class $\left[\widetilde{E_{1}}\right],\left[\widetilde{E_{2}}\right]$ and $\left[\widetilde{E_{m}}\right]$ in ascending sequence, i.e., $\left[\widetilde{E_{3}}\right] \leqslant \sim s\left[\widetilde{E_{1}}\right] \leqslant \sim s$ $\left[\widetilde{E_{2}}\right]$.

Step7: Due to $\left.\mid \widetilde{E_{2}}\right] \mid=2>1$, we used weighted average operator to aggregation element of equivalence class $\left[\widetilde{E_{2}}\right]$. Assume that the weighting vector $T=\left[t_{1}, t_{2}\right]^{T}=[0.4,0.6]$, we can get fuzzy set,

$\widetilde{E_{6}}=0.4 \widetilde{E_{2}} \oplus 0.6 \widetilde{E_{5}}=0.4 \otimes\left(0.14 / s_{0}+0.17 / s_{1}+\right.$ $\left.0.315 / s_{2}+0.285 / s_{3}+0.09 / s_{4}\right) \oplus 0.6 \otimes\left(0.08 / s_{-3}+\right.$ $0.1 / s_{-1}+0.08 / s_{0}+0.06 / s_{1}+0.26 / s_{2}+0.385 / s_{3}+$ $\left.0.155 / s_{4}\right)=0.048 / s_{-3}+0.06 / s_{-1}+0.104 / s_{0}+$ $0.104 / s_{1}+0.282 / s_{2}+0.345 / s_{3}+0.129 / s_{4}$.

According to Proposition 7, $\widetilde{E_{6}} \in\left[\widetilde{E_{2}}\right]$. Since, we show that the better alternative is $x_{2}$ and $x_{5}, \widetilde{E_{6}}$ is the final evaluation value of the better alternatives.

Example 6. Assume that there are five signals $x_{1}$, $x_{2}, x_{3}, x_{4}$ and $x_{5}$, which are represented by five attributes, i.e., the average value analysis (denoted by $f_{1}$ ), the variance analysis (denoted by $f_{2}$ ), the peak one analysis (denoted by $f_{3}$ ), the peak two analysis (denoted by $f_{4}$ ) and the peak three analysis (denoted by $f_{5}$ ) (shown in Table 1 ). There are three experts $D_{1}, D_{2}$ and $D_{3}$ who want to find which on of them is more representative of radar signals. Assume that the weighting vector $H$ of experts is shown as $H=\left[h_{1}, h_{2}, h_{3}\right]^{T}=[0.3,0.4,0.3]^{T}$, where $h_{i}$ denotes the weight of the expert $D_{i}$ and $1 \leqslant i \leqslant 3$. The weighting vector $V$ of the five attributes is shown as $V=\left[v_{1}, v_{2}, v_{3}, v_{4}, v_{5}\right]^{T}=[0.3,0.2,0.25,0.1,0.15]^{T}$, where $v_{i}$ denotes the weight of the attribute $f_{i}$ and $1 \leqslant i \leqslant 5$.

Table 1. Attribute values of five signals

\begin{tabular}{c|ccccc}
\hline & $f_{1}$ & $f_{2}$ & $f_{3}$ & $f_{4}$ & $f_{5}$ \\
\hline$x_{1}$ & 6.81945 & 208.086 & 72.86 & 34.48 & 33.09 \\
$x_{2}$ & -5.04713 & 10.3867 & 21.06 & 10.01 & 7 \\
$x_{3}$ & -6.68135 & 4.06517 & 15.95 & -2.8 & -2.81 \\
$x_{4}$ & -4.84008 & 9.57476 & 21.27 & 8.99 & 6.03 \\
$x_{5}$ & -4.48531 & 41.6989 & 19.34 & 18.73 & 18.15 \\
\hline
\end{tabular}

Experts use five linguistic terms $\left\{s_{-2}, s_{-1}, s_{0}, s_{1}\right.$, $\left.s_{2}\right\}$ to evaluate every signal according to every corresponding to attribute, where $s_{-2}=$ extremely unlike, $s_{-1}=$ unlike, $s_{0}=$ fair, $s_{1}=$ like, $s_{2}=$ extremely like. Formally, to find which on of signals is more representative of radar signals is transformed into a fuzzy multi-criteria decision making problem.

Step1: Construct the fuzzy evaluating matrix $\widetilde{F}_{k}$ for decision- maker $D_{k}$ with respect to attribute $f_{i}$ of the signal $x_{j}$, where $1 \leqslant k \leqslant 3,1 \leqslant i \leqslant 5,1 \leqslant j \leqslant 5$ shown as follows:

$$
\widetilde{F}_{1}=\begin{aligned}
& f_{1} \\
& f_{2} \\
& f_{3} \\
& f_{4} \\
& f_{5}
\end{aligned}\left(\begin{array}{ccccc}
\widetilde{f_{11}^{1}} & \widetilde{f_{12}^{1}} & \widetilde{f_{13}^{1}} & \widetilde{f_{14}^{1}} & \widetilde{f_{15}^{1}} \\
f_{5}^{1} & \widetilde{f_{22}^{1}} & \widetilde{f_{23}^{1}} & \widetilde{f_{24}^{1}} & \widetilde{f_{25}^{1}} \\
\widetilde{f_{41}^{1}} & \widetilde{f_{42}^{1}} & \widetilde{f_{43}^{1}} & \widetilde{f_{34}^{1}} & \widetilde{f_{35}^{1}} \\
\widetilde{f_{51}^{1}} & \widetilde{f_{52}^{1}} & \widetilde{f_{53}^{1}} & \widetilde{f_{54}^{1}} & \widetilde{f_{55}^{1}}
\end{array}\right),
$$




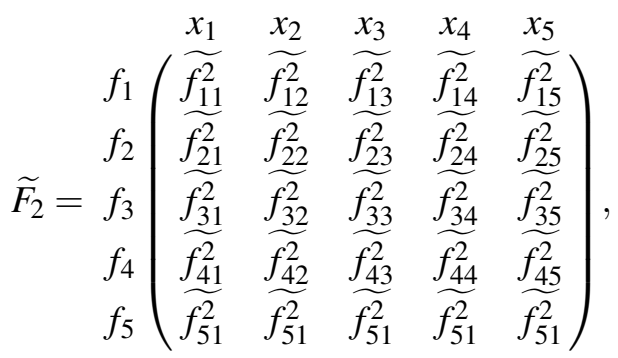

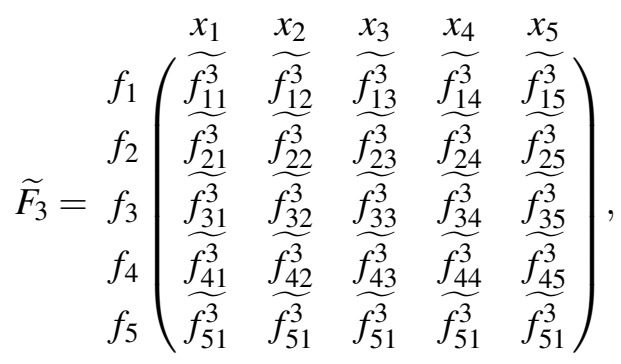

where $\widetilde{f_{11}^{1}}=0.9 / s_{-2}+0.6 / s_{-1}+0.2 / s_{0}, \widetilde{f_{12}^{1}}=$ $0.6 / s_{-2}+0.1 / s_{-1}+0.3 / s_{2}, \quad \widetilde{f_{13}^{1}}=0.9 / s_{-2}+$ $0.8 / s_{-1}+0.4 / s_{0}, \widetilde{f_{14}^{1}}=0.2 / s_{0}+0.2 / s_{1}+0.1 / s_{2}$, $\widetilde{f_{15}^{1}}=0.8 / s_{-2}+0.8 / s_{-1}+0.6 / s_{0}+0.4 / s_{1}, \widetilde{f_{21}^{1}}=$ $0.6 / s_{-2}+0.8 / s_{0}+0.8 / s_{1}, \widetilde{f_{22}^{1}}=0.2 / s_{-1}+0.8 / s_{1}+$ $0.9 / s_{2}, \widetilde{f_{23}^{1}}=0.6 / s_{-2}+0.2 / s_{0}+0.2 / s_{1}+0.4 / s_{2}$, $\widetilde{f_{24}^{1}}=0.6 / s_{0}+0.9 / s_{1}+0.8 / s_{2}, \widetilde{f_{25}^{1}}=0.4 / s_{-2}+$ $0.4 / s_{0}+0.8 / s_{1}+0.9 / s_{2}, \widetilde{f_{31}^{1}}=0.8 / s_{-2}+0.6 / s_{-1}+$ $0.8 / s_{0}, \widetilde{f_{32}^{1}}=0.2 / s_{-1}+0.2 / s_{0}+0.9 / s_{2}, \widetilde{f_{33}^{1}}=$ $0.6 / s_{-2}+0.2 / s_{0}+0.2 / s_{1}+0.4 / s_{2}, \widetilde{f_{34}^{1}}=0.2 / s_{0}+$ $0.8 / s_{1}+0.4 / s_{2}, \widetilde{f_{35}^{1}}=0.8 / s_{-2}+0.8 / s_{-1}+0.5 / s_{1}$, $\widetilde{f_{41}^{1}}=0.8 / s_{-2}+0.6 / s_{-1}+0.3 / s_{1}, \widetilde{f_{42}^{1}}=0.3 / s_{0}+$ $0.8 / s_{1}+0.9 / s_{2}, \widetilde{f_{43}^{1}}=0.3 / s_{-2}+0.2 / s_{-1}, \widetilde{f_{44}^{1}}=$ $0.5 / s_{0}+0.9 / s_{1}+0.9 / s_{2}, \widetilde{f_{45}^{1}}=0.6 / s_{-1}+0.8 / s_{1}+$ $0.4 / s_{2}, \widetilde{f_{51}^{1}}=0.8 / s_{-2}+0.9 / s_{-1}+0.8 / s_{0}, \widetilde{f_{52}^{1}}=$ $0.8 / s_{-2}+0.2 / s_{2}, \widetilde{f_{53}^{1}}=0.8 / s_{-2}+0.7 / s_{-1}, \widetilde{f_{54}^{1}}=$ $0.4 / s_{-2}+0.8 / s_{1}, \widetilde{f_{55}^{1}}=0.2 / s_{-2}+0.3 / s_{-1}+0.2 / s_{1}$, $\widetilde{f_{11}^{2}}=0.9 / s_{-2}+0.1 / s_{2}, \widetilde{f_{12}^{2}}=0.4 / s_{0}+0.8 / s_{1}+$ $0.7 / s_{2}, \widetilde{f_{13}^{2}}=0.9 / s_{-2}+0.8 / s_{-1}, \widetilde{f_{14}^{2}}=0.2 / s_{-2}+$ $0.8 / s_{1}, \widetilde{f_{15}^{2}}=0.8 / s_{-2}+0.8 / s_{-1}+0.6 / s_{0}+0.2 / s_{2}$, $\widetilde{f_{21}^{2}}=0.6 / s_{-2}+0.4 / s_{0}+0.4 / s_{1}, \widetilde{f_{22}^{2}}=0.8 / s_{0}+$ $0.9 / s_{1}+0.9 / s_{2}, \widetilde{f_{23}^{2}}=0.6 / s_{-1}+0.6 / s_{1}+0.2 / s_{2}$, $\widetilde{f_{24}^{2}}=0.9 / s_{1}+0.8 / s_{2}, \widetilde{f_{25}^{2}}=0.8 / s_{0}+0.8 / s_{1}+$ $0.9 / s_{2}, \widetilde{f_{31}^{2}}=0.8 / s_{-2}+0.4 / s_{-1}, \widetilde{f_{32}^{2}}=0.6 / s_{0}+$ $0.8 / s_{1}+0.5 / s_{2}, \widetilde{f_{33}^{2}}=0.6 / s_{-1}+0.6 / s_{1}+0.2 / s_{2}$, $\widetilde{f_{34}^{2}}=0.8 / s_{1}+0.6 / s_{2}, \widetilde{f_{35}^{2}}=0.6 / s_{-2}+0.8 / s_{-1}+$ $0.5 / s_{1}, \widetilde{f_{41}^{2}}=0.4 / s_{-2}+0.7 / s_{1}+0.6 / s_{2}, \widetilde{f_{42}^{2}}=$ $0.7 / s_{0}+0.8 / s_{1}+0.9 / s_{2}, \widetilde{f_{43}^{2}}=0.5 / s_{-2}+0.6 / s_{0}$, $\widetilde{f_{44}^{2}}=0.9 / s_{0}+0.9 / s_{1}+0.9 / s_{2}, \quad \widetilde{f_{45}^{2}}=0.8 / s_{0}+$ $0.8 / s_{1}+0.6 / s_{2}, \widetilde{f_{51}^{2}}=0.8 / s_{-2}+0.7 / s_{-1}+0.8 / s_{1}$, $\widetilde{f_{52}^{2}}=0.6 / s_{0}+0.8 / s_{1}+0.8 / s_{2}, \widetilde{f_{53}^{2}}=0.8 / s_{-2}+$ $0.9 / s_{-1}+0.6 / s_{0}, \widetilde{f_{54}^{2}}=0.8 / s_{0}+0.6 / s_{1}, \widetilde{f_{55}^{2}}=$ $0.5 / s_{-1}, \widetilde{f_{11}^{3}}=0.9 / s_{-2}+0.5 / s_{-1}+0.1 / s_{2}, \widetilde{f_{12}^{3}}=$ $0.4 / s_{0}+0.3 / s_{1}+0.7 / s_{2}, \widetilde{f_{13}^{3}}=0.9 / s_{-2}+0.3 / s_{-1}+$ $0.5 / s_{2}, \widetilde{f_{14}^{3}}=0.2 / s_{-2}+0.8 / s_{1}, \widetilde{f_{15}^{3}}=0.8 / s_{-2}+$ $0.3 / s_{-1}+0.6 / s_{0}+0.7 / s_{2}, \widetilde{f_{21}^{3}}=0.1 / s_{-2}+0.4 / s_{0}+$ $0.9 / s_{1}+0.5 / s_{2}, \widetilde{f_{22}^{3}}=0.8 / s_{0}+0.9 / s_{1}+0.9 / s_{2}$, $\widetilde{f_{23}^{3}}=0.1 / s_{-1}+0.5 / s_{0}+0.1 / s_{1}+0.7 / s_{2}, \widetilde{f_{24}^{3}}=$ $0.5 / s_{0}+0.9 / s_{1}+0.8 / s_{2}, \widetilde{f_{25}^{3}}=0.5 / s_{-1}+0.3 / s_{0}+$ $0.3 / s_{1}+0.4 / s_{2}, \widetilde{f_{31}^{3}}=0.8 / s_{-2}+0.9 / s_{-1}+0.5 / s_{2}$, $\widetilde{f_{32}^{3}}=0.6 / s_{0}+0.8 / s_{1}, \widetilde{f_{33}^{3}}=0.1 / s_{-1}+0.5 / s_{0}+$ $0.1 / s_{1}+0.7 / s_{2}, \widetilde{f_{34}^{3}}=0.5 / s_{0}+0.8 / s_{1}+0.1 / s_{2}$, $\widetilde{f_{35}^{3}}=0.2 / s_{-2}+0.8 / s_{-1}+0.5 / s_{0}, \widetilde{f_{41}^{3}}=0.9 / s_{-2}+$ $0.7 / s_{1}+0.1 / s_{2}, \quad \widetilde{f_{42}^{3}}=0.7 / s_{0}+0.8 / s_{1}+0.4 / s_{2}$, $\widetilde{f_{43}^{3}}=0.1 / s_{0}+0.5 / s_{1}+0.5 / s_{2}, \widetilde{f_{44}^{3}}=0.4 / s_{0}+$ $0.9 / s_{1}+0.9 / s_{2}, \widetilde{f_{45}^{3}}=0.3 / s_{0}+0.8 / s_{1}+0.6 / s_{2}$, $\widetilde{f_{51}^{3}}=0.8 / s_{-2}+0.7 / s_{-1}+0.8 / s_{1}, \widetilde{f_{52}^{3}}=0.1 / s_{0}+$ $0.8 / s_{1}+0.3 / s_{2}, \widetilde{f_{53}^{3}}=0.8 / s_{-2}+0.9 / s_{-1}+0.1 / s_{0}$, $\widetilde{f_{54}^{3}}=0.8 / s_{0}+0.1 / s_{1}+0.5 / s_{2}, \widetilde{f_{55}^{3}}=0.2 / s_{-2}+$ $0.5 / s_{-1}+0.5 / s_{1}$.

Step2: Assume that the weighting vector $W=$ $\left[w_{1}, w_{2}, w_{3}\right]^{T}=[0.5,0.3,0.2]^{T}$. The weight $\widetilde{Z_{i j}}$ of the attribute $f_{i}$ of the signal $x_{j}$ is calculated by the FIOWA operators, i.e.,

$$
\begin{aligned}
\widetilde{Z_{i j}} & =F_{\text {FIOWA }}\left(\left\langle h_{1}, \widetilde{f_{i j}^{1}}\right\rangle,\left\langle h_{2}, \widetilde{f_{i j}^{2}}\right\rangle,\left\langle h_{3}, \widetilde{f_{i j}^{3}}\right\rangle\right) \\
& =w_{1} \widetilde{f_{i j}^{\prime}} \oplus w_{2} \widetilde{f_{i j}^{\prime 2}} \oplus w_{3} \widetilde{f_{i j}^{\prime 3}},
\end{aligned}
$$

where $h_{k}$ denotes the weighting vector of decisionmakers $D_{k}, \quad 1 \leqslant i \leqslant 5,1 \leqslant j \leqslant 5,1 \leqslant k \leqslant$ 
3, $\widetilde{f_{i j}^{\prime} k}$ is the value of the OWA pair having the $k$ th largest order inducing $h$ value. The results are shown by $\widetilde{Z_{11}}=0.9 / s_{-2}+0.4 / s_{-1}+$ $0.1 / s_{0}+0.05 / s_{2}, \widetilde{Z_{21}}=0.5 / s_{-2}+0.6 / s_{0}+0.7 / s_{1}+$ $0.1 / s_{2}, \widetilde{Z_{31}}=0.8 / s_{-2}+0.6 / s_{-1}+0.4 / s_{0}+0.1 / s_{2}$, $\widetilde{Z_{41}}=0.7 / s_{-2}+0.3 / s_{-1}+0.5 / s_{1}+0.2 / s_{2}, \widetilde{Z_{51}}=$ $0.8 / s_{-2}+0.8 / s_{-1}+0.4 / s_{0}+0.4 / s_{1}$,

$\widetilde{Z_{12}}=0.3 / s_{-2}+0.05 / s_{-1}+0.2 / s_{0}+0.3 / s_{1}+$ $0.5 / s_{2}, \widetilde{Z_{22}}=0.1 / s_{-1}+0.4 / s_{0}+0.85 / s_{1}+0.9 / s_{2}$ $\widetilde{Z_{32}}=0.1 / s_{-1}+0.4 / s_{0}+0.4 / s_{1}+0.6 / s_{2}, \widetilde{Z_{42}}=$ $0.5 / s_{0}+0.8 / s_{1}+0.8 / s_{2}, \widetilde{Z_{52}}=0.4 / s_{-2}+0.2 / s_{0}+$ $0.4 / s_{1}+0.4 / s_{2}$,

$\widetilde{Z_{13}}=0.9 / s_{-2}+0.7 / s_{-1}+0.2 / s_{0}+0.1 / s_{2}$, $\widetilde{Z_{23}}=0.3 / s_{-2}+0.2 / s_{-1}+0.2 / s_{0}+0.3 / s_{1}+0.4 / s_{2}$, $\widetilde{Z_{33}}=0.8 / s_{-2}+0.6 / s_{-1}+0.2 / s_{0}, \widetilde{Z_{43}}=0.3 / s_{-2}+$ $0.1 / s_{-1}+0.2 / s_{0}+0.1 / s_{1}+0.1 / s_{2}, \widetilde{Z_{53}}=0.8 / s_{-2}+$ $0.8 / s_{-1}+0.2 / s_{0}, \widetilde{Z_{14}}=0.1 / s_{-2}+0.1 / s_{0}+0.5 / s_{1}+$ $0.05 / s_{2}, \widetilde{Z_{24}}=0.4 / s_{0}+0.9 / s_{1}+0.8 / s_{2}, \widetilde{Z_{34}}=$ $0.2 / s_{0}+0.8 / s_{1}+0.4 / s_{2}, \widetilde{Z_{44}}=0.6 / s_{0}+0.9 / s_{1}+$ $0.9 / s_{2}, \widetilde{Z_{54}}=0.2 / s_{-2}+0.4 / s_{0}+0.6 / s_{1}+0.1 / s_{2}$, $\widetilde{Z_{15}}=0.8 / s_{-2}+0.7 / s_{-1}+0.6 / s_{0}+0.2 / s_{1}+0.2 / s_{2}$, $\widetilde{Z_{25}}=0.2 / s_{-2}+0.1 / s_{-1}+0.5 / s_{0}+0.7 / s_{1}+0.8 / s_{2}$, $\widetilde{Z_{35}}=0.6 / s_{-2}+0.8 / s_{-1}+0.4 / s_{0}+0.2 / s_{1}, \widetilde{Z_{45}}=$ $0.3 / s_{-1}+0.3 / s_{0}+0.8 / s_{1}+0.6 / s_{2}, \widetilde{Z_{55}}=0.2 / s_{-2}+$ $0.4 / s_{-1}+0.2 / s_{1}$.

Step3: Assume that the weighting vector $R=\left[r_{1}, r_{2}, r_{3}, r_{4}, r_{5}\right]^{T}=[0.3,0.25,0.15,0.1]^{T}$, by using FIOWA operator, we get the evaluation value $\widetilde{E}_{j}$ of signal $x_{j}$, i.e., $\widetilde{E}_{j}=$ $F_{\text {FIOWA }}\left(\left\langle v_{1}, \widetilde{Z_{1 j}}\right\rangle,\left\langle v_{2}, \widetilde{Z_{2 j}}\right\rangle,\left\langle v_{3}, \widetilde{Z_{3 j}}\right\rangle,\left\langle v_{4}, \widetilde{Z_{4 j}}\right\rangle,\left\langle v_{5}, \widetilde{Z_{5 j}}\right\rangle\right)$ $=r_{1}{\widetilde{Z_{1 j}}}^{\prime} \oplus r_{2}{\widetilde{Z_{2 j}}}^{\prime} \oplus r_{3}{\widetilde{Z_{3 j}}}^{\prime} \oplus r_{4}{\widetilde{Z_{4 j}}}^{\prime} \oplus r_{5}{\widetilde{Z_{5 j}}}^{\prime}, v_{i}$ denotes the weight of attribute $f_{i},{\widetilde{Z_{i j}}}^{\prime}$ is the value of the OWA pair having the $i$ th largest order inducing $v$ value, $1 \leqslant i \leqslant 5,1 \leqslant j \leqslant 5$. The results are shown by $\widetilde{E_{1}}=0.76 / s_{-2}+0.42 / s_{-1}+$ $0.31 / s_{0}+0.25 / s_{1}+0.08 / s_{2}, \widetilde{E_{2}}=0.15 / s_{-2}+$ $0.06 / s_{-1}+0.32 / s_{0}+0.47 / s_{1}+0.62 / s_{2}, \widetilde{E_{3}}=$ $0.68 / s_{-2}+0.53 / s_{-1}+0.2 / s_{0}+0.07 / s_{1}+0.12 / s_{2}$, $\widetilde{E_{4}}=0.06 / s_{-2}+0.28 / s_{0}+0.71 / s_{1}+0.38 / s_{2}$ and $\widetilde{E_{5}}=0.46 / s_{-2}+0.52 / s_{-1}+0.41 / s_{0}+0.36 / s_{1}+$ $0.28 / s_{2}$.

Step4: Based on (1), the score $S\left(\widetilde{E}_{i} \ominus \widetilde{E}_{j}\right)$ of the weighted difference of membership values between
$\widetilde{E}_{i}$ and $\widetilde{E}_{j}$ is calculated, where $1 \leqslant j \leqslant 5$. The results are shown by $S\left(\widetilde{E_{1}} \ominus \widetilde{E_{2}}\right)=-2.88, S\left(\widetilde{E_{1}} \ominus \widetilde{E_{3}}\right)=$ $0.05, S\left(\widetilde{E_{1}} \ominus \widetilde{E_{4}}\right)=-2.88, S\left(\widetilde{E_{1}} \ominus \widetilde{E_{5}}\right)=-1.11$, $S\left(\widetilde{E_{2}} \ominus \widetilde{E_{3}}\right)=2.93, S\left(\widetilde{E_{2}} \ominus \widetilde{E_{4}}\right)=0, S\left(\widetilde{E_{2}} \ominus \widetilde{E_{5}}\right)=$ $1.87, S\left(\widetilde{E_{3}} \ominus \widetilde{E_{4}}\right)=-2.93, S\left(\widetilde{E_{3}} \ominus \widetilde{E_{5}}\right)=-1.06$, $S\left(\widetilde{E_{4}} \ominus \widetilde{E_{5}}\right)=1.87$.

Step5: All evaluations of alternatives are classified by the equivalence relation $\sim_{s}$, therefore, we have $\left[\widetilde{E_{1}}\right]=\left\{\widetilde{E_{1}}\right\},\left[\widetilde{E_{2}}\right]=\left\{\widetilde{E_{2}}, \widetilde{E_{4}}\right\},\left[\widetilde{E_{3}}\right]=\left\{\widetilde{E_{3}}\right\}$, $\left[\widetilde{E_{4}}\right]=\left\{\widetilde{E_{5}}\right\}$.

Step6: Sort the equivalence class $\left[\widetilde{E_{1}}\right],\left[\widetilde{E_{2}}\right],\left[\widetilde{E_{3}}\right]$ and $\left[\widetilde{E_{4}}\right]$ in ascending sequence, $\left[\widetilde{E_{3}}\right] \leqslant \sim s\left[\widetilde{E_{1}}\right] \leqslant \sim s$ $\left[\widetilde{E_{4}}\right] \leqslant \sim s\left[\widetilde{E_{2}}\right]$.

Step7: Due to $\left.\mid \widetilde{E_{2}}\right] \mid=2>1$, we used weighted average operator to aggregation element of equivalence class $\left[\widetilde{E_{2}}\right]$ and average operator to aggregation each feature of signals $x_{2}$ and $x_{4}$, which are shown in Table 2. Assume that the weighting vector $T=\left[t_{1}, t_{2}\right]^{T}=[0.5,0.5]$. We can get the new signal $x_{6}$ which can be used to instead of signals $x_{2}$ and $x_{4}$, Its evaluation value is the fuzzy set $\widetilde{E_{6}}$, i.e., $\widetilde{E_{6}}=0.5 \widetilde{E_{2}} \oplus 0.5 \widetilde{E_{4}}=0.5 \otimes\left(0.15 / s_{-2}+0.06 / s_{-1}+\right.$ $\left.0.32 / s_{0}+0.47 / s_{1}+0.62 / s_{2}\right) \oplus 0.5 \otimes\left(0.06 / s_{-2}+\right.$ $\left.0.28 / s_{0}+0.71 / s_{1}+0.38 / s_{2}\right)=0.105 / s_{-2}+$ $0.03 / s_{-1}+0.3 / s_{0}+0.59 / s_{1}+0.5 / s_{2}$.

Table 2. Attribute values of the signal $x_{6}$

\begin{tabular}{c|ccccc}
\hline & $f_{1}$ & $f_{2}$ & $f_{3}$ & $f_{4}$ & $f_{5}$ \\
\hline$x_{6}$ & -4.943605 & 9.98073 & 21.165 & 9.5 & 6.515 \\
\hline
\end{tabular}

According to Proposition $7, \widetilde{E_{6}} \in\left[\widetilde{E_{2}}\right] \cdot x_{6}$ is representative of radar signals.

\section{Conclusion}

In this paper, we analyze some algebraic properties of the score $S\left(\widetilde{P}_{i} \ominus \widetilde{P}_{j}\right)$ and prove that the order relation decided by the score is a pre-order relation of fuzzy sets on $U$. Then, we provide an equivalence relation on fuzzy sets based on $S\left(\widetilde{P}_{i} \ominus \widetilde{P}_{j}\right)$ and propose a new method to handle fuzzy group decisionmaking. Some numerical example illustrates our method can be used to improve the best alternative of fuzzy group decision-making when its ordering is pre-ordering. 


\section{Acknowledgments}

This work is partially supported by the national natural science foundation of China (61372187), Sichuan Key Technology Research and Development Program (2012GZ0019, 2013GXZ0155) and the open research fund of key laboratory of intelligent network information processing, Xihua University (SZJJ2013-020).

\section{References}

1. K.T. Atanassov, Intuitionistic fuzzy sets, Fuzzy Sets and Systems, 20, 87-96 (1986).

2. T.Y. Chen, H.P. Wang and Y.Y. Lu, A multicriteria group decision-making approach based on intervalvalued intuitionistic fuzzy sets: A comparative perspective, Expert Systems with Applications, 38(5), 7647-7658 (2011).

3. S.M. Chen and S.J. Niou, Fuzzy multiple attributes group decision-making based on fuzzy induced OWA operators, Expert Systems with Applications, 38(4), 4097-4108 (2011).

4. S.J. Chen and S.M. Chen, A new method for handling multicriteria fuzzy decision making problems using FN-IOWA operators, Cybernetics \& Systems, 34(2), 109-137 (2011).

5. F. Chiclana, E. Herrera-Viedma, F. Herrera and S. Alonso, Some induced ordered weighted averaging operators and their use for solving group decisionmaking problems based on fuzzy preference relations, European Journal of Operational Research, 182(1), 383-399 (2007).

6. D. Dubois, H. Prade, The three semantics of fuzzy sets, Fuzzy Sets and Systems, 90, 141-150 (1997).

7. J. Fortin, D. Dubois and H. Fargier, Gradual numbers and their application to fuzzy interval analysis, IEEE Transactions on Fuzzy Systems, 16(2), 388-402 (2008).

8. F. Herrera and L. Martínez, A 2-tuple fuzzy linguistic representation model for computing with words, IEEE Transactions on Fuzzy Systems, 8, 746-752 (2000).

9. F. Herrera, E. Herrera-Viedma and L. Martínez, A fusion approach for managing multi-granularity linguistic term sets in decision making, Fuzzy Sets and Systems, 114, 43-58 (2000).

10. D.H. Hong and C.H. Choi, Multicriteria fuzzy decision-making problems based on vague set theory, Fuzzy Sets and Systems, 114, 103-113 (2000).

11. D.F. Li, Compromise ratio method for fuzzy multiattribute group decisio making, Applied Soft Computing Journal, 7(3), 807-817 (2007).
12. D.F. Li, The GOWA operator based approach to multiattribute decision making using intuitionistic fuzzy sets, Mathematical and Computer Modelling, 53, 1182-1196 (2011).

13. C.J. Lin and W.W. Wu, A causal analytical method for group decision-making under fuzzy environment, Expert Systems with Applications, 34(1), 205-213 (2008).

14. L. Martínez, F. Herrera, An overview on the 2-tuple linguistic model for computing with words in decision making: Extensions, applications and challenges, Information Sciences, 207, 1-18 (2012).

15. L. Martínez, Sensory evaluation based on linguistic decision analysis, International Journal of Approximate Reasoning, 44(2), 148-164 (2007).

16. L. Martínez, J. Liu, D. Ruan and J.B. Yang, Dealing with heterogeneous information in engineering evaluation processes, Information Sciences, 177(7), 15331542 (2007).

17. F. Mata, L. Martínez, E. Herrera-Viedma, An adaptive consensus support model for group decision-making problems in a multigranular fuzzy linguistic context, IEEE Transactions on Fuzzy Systems, 17(2), 279-290 (2009).

18. N.R. Pal, et al, Uncertainties with Atanassov's intuitionistic fuzzy sets: Fuzziness and lack of knowledge, Information Sciences, 228, 61-74 (2013).

19. Z. Pei, D. Ruan, J. Liu and Y. Xu, Linguistic Values based Intelligent Information Processing: Theory, Methods, and Application, Atlantis press \& World Scientific, 2009.

20. Z. Pei, D. Ruan, J. Liu and Y. Xu, A linguistic aggregation operator with three kinds of weights for nuclear safeguards evaluation, Knowledge-Based Systems, 28, 19-26 (2012).

21. Z. Pei, P. Shi, Fuzzy risk analysis based on linguistic aggregation operators, International Journal of Innovative Computing, Information and Control, 7(12), 7105-7118 (2011).

22. R.M. Rodríguez, L. Martínez and F. Herrera, Hesitant fuzzy linguistic term sets for decision making, IEEE Transactions on Fuzzy Systems, 20(1), 109-119 (2012).

23. F. Wang, C. Zhang and Z. Xia, Equivalence of the cut sets-based decomposition theorems and representation theorems on intuitionistic fuzzy sets and intervalvalued fuzzy sets, Mathematical and Computer Modelling, 57, 1364-1370 (2013).

24. C.F. Wei, Z. Pei and H. M. Li, An induced OWA operator in coal mine safety evaluation, Journal of Computer and System Sciences, 78, 997-1005 (2012).

25. R.R. Yager, On ordered weighted averaging aggregation operators in multicriteria decision making, IEEE Transactions on Systems Man and Cybernetics, 18(1), 183-190 (1988). 
26. R.R. Yager, D. P. Filev, Induced ordered weighted averaging operators, IEEE Transactions on Systems, Man, and Cybernetics-Part B: Cybernetics, 29(2), 141-150 (1999).

27. R.R. Yager, Some aspects of intuitionistic fuzzy sets, Fuzzy Optimization and Decision Making, 8, 67-90 (2009).
28. X.H. Yuan, H.X. Li, K.B. Sun, The cut sets, decomposition theorems and representation theorems on intuitionistic fuzzy sets and interval valued fuzzy sets, Science China (Information Sciences), 54(1), 91-110 (2011).

29. L. A. Zadeh, Fuzzy sets, Information and Control, 8(3), 338-353 (1965). 